\title{
Anti-windup for a class of partially linearisable nonlinear systems with application to Wave Energy Converter control
}

\author{
Angeliki Lekka ${ }^{1 *}$, Matthew C. Turner ${ }^{1}$, Prathyush P. Menon ${ }^{2}$ \\ ${ }^{1}$ Control Systems Research Group, University of Leicester, Leicester, LE1 7RH, UK \\ ${ }^{2}$ Centre for Systems, Dynamics and Control, College of Engineering, Mathematics and Physical \\ Sciences, University of Exeter, Exeter, EX4 4QF, UK \\ *al385@le.ac.uk
}

\begin{abstract}
This paper studies the anti-windup problem for a certain class of nonlinear systems, in which the plant is globally quadratically stable and also partially linearisable by a suitably chosen nonlinear feedback control law. Three types of anti-windup compensator are proposed for this type of nonlinear system: the first is a nonlinear extension of the popular linear Internal Model Control scheme; the second has a similar structure to the IMC anti-windup compensator yet is of reduced order and has entirely linear dynamics; the third is again a linear anti-windup compensator but can bestow the closed-loop system with some sub-optimal performance properties. All three anti-windup compensators are able to provide global exponential stability guarantees for the aforementioned class of systems. This work was inspired by a wave energy application whose dynamics fall into the class of systems studied in the paper. Simulation results show the efficacy of the three AW compensators when applied to the wave energy application.
\end{abstract}

\section{Introduction}

Anti-windup (AW) compensation is a common approach of ensuring a system's behaviour remains acceptable if the demanded control signal exceeds the control constraints present in the system. Despite having its origins in industry, the past two decades have seen the development of numerous approaches to enable engineers to design anti-windup compensators with stability and performance guarantees - the reader is referred, in particular, to the surveys [1,2] or the books [3-6] and references therein. Traditionally, anti-windup design has been focused on linear plants and linear controllers; this has great practical appeal because many plants/controllers may be reasonably well modelled with linear approximations. Furthermore, for this class of systems, it is relatively straightforward to harness the power of modern convex optimisation techniques for AW analysis and design [7-9]; alternatively, frequency domain tools may be deployed for a graphical assessment of stability [10-12].

For systems that are not easy to approximate as linear, AW design becomes more complicated; the stability and performance properties cannot be easily inferred and it is unlikely that one common AW all-encompassing technique will be developed. Consequently, researchers have considered special classes of nonlinear systems and have developed anti-windup techniques centred on these individual classes. An early reference which proposes a rigorous AW synthesis method for a class of nonlinear Euler-Lagrange systems is [13], where the techniques introduced in [14] are adapted. Similarly, [15] modified the AW approach in [16] for a class of indirect adaptive control 
systems. Recently, a differential algebraic representation of nonlinear systems has been proposed ( [17]) and applied to the AW problem for nonlinear systems [18]. The merit of [18] is that various concepts used in linear AW are generalised, but the transformation proposed is rather complicated and the AW synthesis conditions are typically non-convex. Furthermore, recent results on constrained control for systems with sector bounded nonlinearities have also been specialised to AW design $[19,20]$.

In addition to the above approaches, the literature has documented the development of AW schemes for feedback linearisable systems [21-25]. Reference [24] developed an anti-windup scheme for a particular class of nonlinear systems controlled, nominally, via a nonlinear dynamic inversion (NDI) controller; parallels between this and the corresponding linear scheme in [26] were observed, and the work of [24] could be considered as a generalisation of [26] to a certain class of nonlinear systems. A key limitation of the results in [24] is the Lipschitz assumption made on the nonlinearity; a further limitation is the assumption that the nonlinearity appears in the range space of the input distribution matrix. The AW construction described in [24] also rests on the solution of a partial differential inequality, which is, in general, not straightforward to obtain.

The work in this paper is motivated by saturation problems in a wave energy converter (WEC) used for potable water production [27,28]. The WEC dynamics are nonlinear and several linear anti-windup schemes which were initially tested in simulation did not work successfully. The dynamics also do not belong to the class of nonlinear systems considered in [24], which gives synthesis conditions for nonlinear AW compensators. However, the plant in [27] has a similar structure to the one in [24] and the controller is a partial-NDI (Nonlinear Dynamic Inversion) type in that it "cancels" some, but not all of the nonlinear terms. The plant consists of two predominantly linear subsystems coupled by two nonlinear terms, one of which is not Lipschitz. This paper proposes three AW compensators for this class of systems: The first two are nonlinear versions of so-called Internal Model Control (IMC) anti-windup, which is well known to be globally stabilising in the linear case. The third contains a free "state-feedback" matrix, which can be used to optimise performance and is one of the key advancements of this work over [29]. This last compensator has a similar structure to that in [24], but its construction requires the solution of a Linear Matrix Inequality (LMI) rather than a nonlinear partial differential inequality as in [24].

This paper introduces three types of anti-windup compensator for the aforementioned class of partially linearisable systems and shows that these compensators provide global exponential stability for the overall closed-loop nonlinear system. It will be seen that all three of the compensators are relatively simple to construct; the IMC compensators require no optimisation and the third compensator requires only a standard convex optimisation. The paper also provides simulation results demonstrating the effectiveness of the techniques on the motivating WEC control problem.

\section{Nominal plant and controller}

\subsection{Notation and preliminaries}

$\|()$.$\| denotes the Euclidean norm of a vector or the norm induced by the Euclidean vector norm$ of a matrix (maximum singular value). $X>0(X<0)$ means the matrix $X$ is positive (negative) definite. $M^{\prime}$ denotes the transpose of a matrix $M \cdot \operatorname{diag}\left(X_{1}, X_{2}, \ldots, X_{n}\right)$ means a block diagonal matrix formed from the matrices $X_{1}, X_{2}, \ldots, X_{n}$ in order. $I_{q}$ denotes the identity matrix of dimension $q \times q$. The decentralised saturation function is defined as 


$$
\operatorname{sat}(u)=\left[\operatorname{sat}_{1}\left(u_{1}\right), \quad \ldots, \quad \operatorname{sat}_{2}\left(u_{m}\right)\right]^{\prime}
$$

where $\operatorname{sat}_{i}\left(u_{i}\right)=\min \left\{\left|u_{i}\right|, \bar{u}_{i}\right\}$. The deadzone function is defined as

$$
\operatorname{Dz}(u)=u-\operatorname{sat}(u)
$$

The saturation and deadzone functions inhabit the sector $[0, I]$ and thus the following fact applies:

Fact 1. Let $\phi(v) \in$ Sector $[0, I]$ where $v \in \mathbb{R}^{m}$ and $\phi():. \mathbb{R}^{m} \mapsto \mathbb{R}^{m}$.

Then for any diagonal $W>0$ the following inequality holds

$$
\phi(v)^{\prime} W[v-\phi(v)] \geq 0 \quad \forall v \in \mathbb{R}^{m}
$$

\subsection{Nominal plant and controller}

2.2.1. Plant: The class of systems considered are given by the following state-space equations:

$$
\mathcal{P} \sim\left\{\begin{aligned}
\dot{x}_{1} & =A_{11} x_{1}+A_{12}\left(x_{1}\right) x_{2}+B_{11} d \\
\dot{x}_{2} & =A_{21}\left(x_{1}\right)+A_{22} x_{2}+B_{21} d+B_{22} u_{m} \\
y & =C_{2} x_{2}
\end{aligned}\right.
$$

where $x_{1} \in \mathbb{R}^{n_{1}}, x_{2} \in \mathbb{R}^{n_{2}}$ and $n=n_{1}+n_{2}$ is the total state dimension of the plant. $d \in \mathbb{R}^{n_{d}}$ represents a disturbance vector, $u_{m}=\operatorname{sat}\left(y_{c}\right) \in \mathbb{R}^{m}$ represents the control input to the plant with $y_{c}$ the output of the controller (to be defined shortly), and $y \in \mathbb{R}^{p}$ represents the output of the system which is available to the linear part of the controller. It is convenient to denote $x:=\left[\begin{array}{ll}x_{1}^{\prime}, & x_{2}^{\prime}\end{array}\right]^{\prime}$. The term $A_{12}():. \mathbb{R}^{n_{1}} \mapsto \mathbb{R}^{n_{1} \times n_{2}}$ represents an "unmatched" nonlinearity which is not in the range space of the input distribution matrix $B_{22}$. The term $A_{21}():. \mathbb{R}^{n_{1}} \mapsto \mathbb{R}^{n_{2}}$ is the "matched" nonlinearity as formalised below.

Assumption 1. Consider the plant $\mathcal{P}$ given by equation (1). It is assumed that:-

1. When $d \equiv 0$ and $u_{m} \equiv 0, \mathcal{P}$ is quadratically stable, that is there exists a symmetric positive definite matrix $P>0$ and a scalar $\alpha>0$ such that with $V(x)=\alpha x^{\prime} P x$ there exists a scalar $\epsilon>0$ such that $\dot{V}(x)<-\alpha \epsilon\|x\|^{2}$ for all $x \in \mathbb{R}^{n}$

2. The matrices $A_{11}$ and $A_{22}$ are Hurwitz.

3. $A_{21}(z)=B_{22} f_{1}(z)$ where $f_{1}():. \mathbb{R}^{n_{1}} \mapsto \mathbb{R}^{m}$ is globally Lipschitz with constant $k_{1}$.

4. $\left\|A_{12}(z)\right\| \leq \beta \quad \forall z \in \mathbb{R}^{n_{1}}$.

Remark 1. The first item ensures that the open-loop plant is globally exponentially stable and parallels the assumption made in the linear case; it is not a necessary requirement (unlike the linear case) but simplifies the presentation of the results. The assumption that $A_{11}$ and $A_{22}$ are Hurwitz is stronger than necessary but is compatible with the form of partial-NDI controller introduced next. The third and fourth items essentially bound the size of the two nonlinear terms under consideration and are structural requirements. 
2.2.2. Controller: The state-space realisation of the controller is given below.

$$
\mathcal{C} \sim\left\{\begin{array}{l}
\dot{x}_{c}=A_{c} x_{c}+B_{c}\left(y-\theta_{2}\right)+B_{c r} r \\
y_{c}=C_{c} x_{c}+D_{c}\left(y-\theta_{2}\right)+D_{c r} r-f_{1}\left(x_{1}\right)+\theta_{1}
\end{array}\right.
$$

where $x_{c} \in \mathbb{R}^{n_{c}}$ is the controller's state, $r \in \mathbb{R}^{n_{r}}$ is the reference and $\theta_{1} \in \mathbb{R}^{m}$ and $\theta_{2} \in \mathbb{R}^{p}$ are the compensation signals generated by the anti-windup compensator; when no saturation is present, $\theta_{1} \equiv 0$ and $\theta_{2} \equiv 0$. Besides the knowledge of the system output $y$, knowledge of either the state $x_{1}$, or measurement of the function $f_{1}\left(x_{1}\right)$ is also assumed. This allows the controller to partially (and only partially) "cancel" the nonlinear terms present.

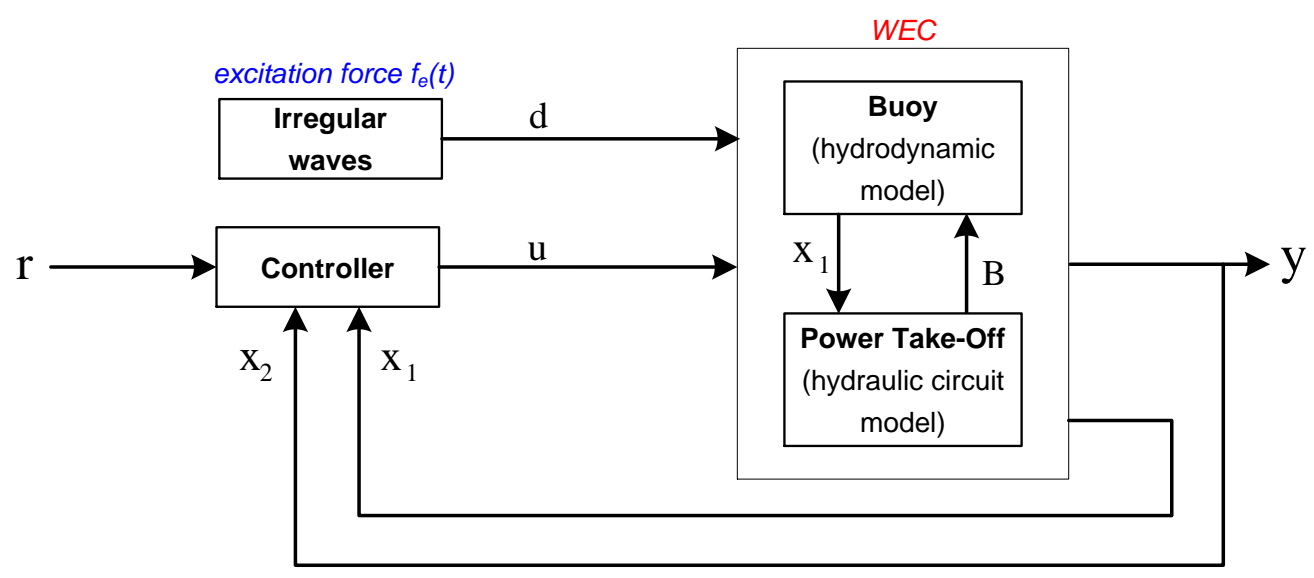

Fig. 1: Block diagram of the system implemented in this paper.

2.2.3. Closed-loop system: Without anti-windup $\left(\theta_{1} \equiv 0\right.$ and $\left.\theta_{2} \equiv 0\right)$, without saturation $\left(u_{m}=y_{c}\right)$ and with the reference, $r$, and disturbance, $d$, set to zero, equations (1) and (2) yield the closed-loop dynamics

$$
\left\{\begin{array}{c}
\dot{x}_{1} \\
{\left[\begin{array}{c}
\dot{x}_{2} \\
\dot{x}_{c}
\end{array}\right]=\left[\begin{array}{cc}
A_{22}+B_{22} D_{c} C_{2} & B_{22} C_{c} \\
B_{c} C_{2} & A_{c}
\end{array}\right]\left[\begin{array}{c}
x_{2} \\
x_{c}
\end{array}\right]}
\end{array}\right.
$$

This can be written more concisely as

$$
\left[\begin{array}{c}
\dot{x}_{1} \\
\dot{x}_{2 c}
\end{array}\right]=\left[\begin{array}{cc}
A_{11} & 0 \\
0 & A_{2 c}
\end{array}\right]\left[\begin{array}{c}
x_{1} \\
x_{2 c}
\end{array}\right]+\left[\begin{array}{c}
A_{12}\left(x_{1}\right) x_{2} \\
0
\end{array}\right]
$$

where $x_{2 c}:=\left[\begin{array}{ll}x_{2}^{\prime} & x_{c}^{\prime}\end{array}\right]^{\prime}$ and

$$
A_{2 c}:=\left[\begin{array}{cc}
A_{22}+B_{22} D_{c} C_{2} & B_{22} C_{c} \\
B_{c} C_{2} & A_{c}
\end{array}\right]
$$

This motivates the second assumption given below.

Assumption 2. The matrix $A_{2 c}$ is Hurwitz 
This assumption is a necessary and sufficient (see Ref [30]) condition for closed-loop stability in the absence of saturation.

\section{Anti-windup design}

\subsection{Nonlinear IMC}

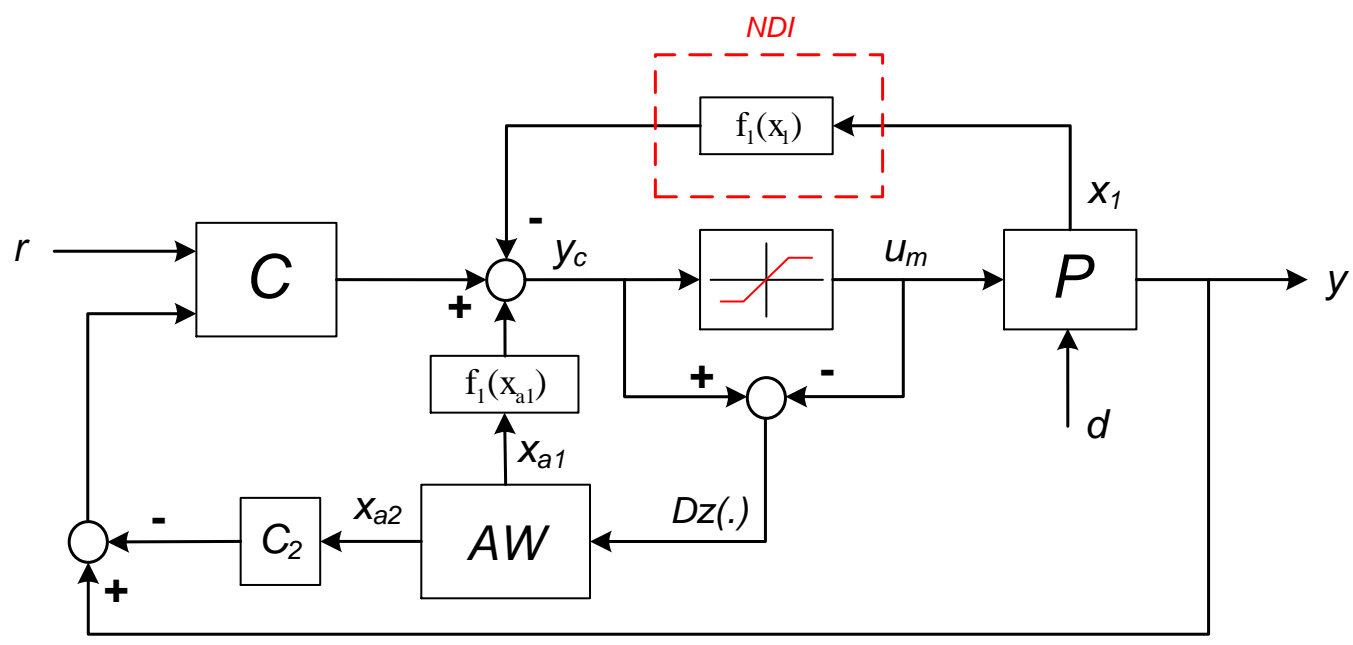

Fig. 2: System block diagram of the proposed nonlinear IMC anti-windup compensator.

The anti-windup compensator, similar to the nonlinear IMC compensator in [24], is a copy of the plant augmented with a state-feedback term (Fig. 2), viz:

$$
\mathcal{A} \mathcal{W}_{F} \sim\left\{\begin{aligned}
\dot{x}_{a 1} & =A_{11} x_{a 1}+A_{12}\left(x_{a 1}\right) x_{a 2} \\
\dot{x}_{a 2} & =A_{21}\left(x_{a 1}\right)+A_{22} x_{a 2}-B_{22} \mathrm{Dz}\left(y_{c}\right) \\
\theta_{1} & =f_{1}\left(x_{a 1}\right) \\
\theta_{2} & =C_{2} x_{a 2}
\end{aligned}\right.
$$

where $x_{a}=\left[\begin{array}{ll}x_{a 1}^{\prime} & x_{a 2}^{\prime}\end{array}\right]^{\prime}$ is the anti-windup state vector, with $x_{a 1} \in \mathbb{R}^{n_{1}}$ and $x_{a 2} \in \mathbb{R}^{n_{2}}$. Standard linear AW compensators are driven by the deadzone function $\operatorname{Dz}(u)=u-\operatorname{sat}(u)$ and become active when saturation occurs; the nonlinear IMC compensator above follows the same operating principle.

The first objective is to show that the dynamics of the overall closed-loop system comprising the anti-windup compensator (6), the plant (1) and the controller (2) remain internally stable when the reference and disturbance inputs are set to zero. To achieve this, a coordinate transformation similar to [22,24], is performed. Let $r \equiv 0$ and $d \equiv 0$ and $u_{m}=\operatorname{sat}\left(y_{c}\right)$ and define $e_{1}:=x_{1}-x_{a 1}$, $e_{2}:=x_{2}-x_{a 2}$ and $e_{2 c}:=\left[\begin{array}{ll}e_{2}^{\prime} & x_{c}^{\prime}\end{array}\right]^{\prime}$. The closed-loop interconnection of the plant (1), controller (2) and anti-windup compensator (6) can then be described in the $\left(e_{1}, e_{2 c}, x_{a 1}, x_{a 2}\right)$ coordinates as follows: 


$$
\left\{\begin{aligned}
\dot{e}_{1} & =A_{11} e_{1}+A_{12}\left(x_{1}\right) x_{2}-A_{12}\left(x_{a 1}\right) x_{a 2} \\
\dot{e}_{2 c} & =A_{2 c} e_{2 c} \\
\dot{x}_{a 1} & =A_{11} x_{a 1}+A_{12}\left(x_{a 1}\right) x_{a 2} \\
\dot{x}_{a 2} & =A_{21}\left(x_{a 1}\right)+A_{22} x_{a 2}-B_{22} \mathrm{Dz}\left(y_{c}\right) \\
y_{c} & =C_{2 c} e_{2 c}-f_{1}\left(x_{1}\right)+f_{1}\left(x_{a 1}\right)
\end{aligned}\right.
$$

where $C_{2 c}=\left[\begin{array}{ll}C_{2} D_{c} & C_{c}\end{array}\right]$.

The main result of the section is the following.

Proposition 1. Consider the interconnection of (1), (2) and (6) through $u_{m}=\operatorname{sat}\left(y_{c}\right)$. Let $r \equiv 0$, $d \equiv 0$ and let Assumptions 1 and 2 be satisfied. Then the origin of the interconnected system is globally exponentially stable.

Proof: See appendix, Section 8.1.

Remark 1. The IMC compensator (6) shares several features with its linear counterpart (see [32]). In particular, it features the same number of states as present in the plant and, hence, may be computationally expensive to implement.

\subsection{Reduced-order IMC}

The partial NDI controller (2) works, under nominal conditions, by cancelling the nonlinear terms in the $x_{2}$ state equation, thereby making the $x_{1}$ portion of the state vector unobservable from the output $y$. The linear part of the controller is then chosen to bestow desirable properties on the $x_{2}$ dynamics. Due to the stability of the $x_{1}$ subsystem, this part of the plant will remain well-behaved and not interfere with the system's operation [30].

When saturation is present, it is impossible to decouple the two subsystems completely, but it does suggest that focusing anti-windup design only on the $x_{2}$ subsystem could be beneficial. Therefore, consider a reduced-order anti-windup compensator with the $x_{a 1}$ state equation omitted and $x_{a 1}$, and functions thereof, set to zero wherever else they appear. In this case, the AW dynamics are

$$
\mathcal{A W}_{R O} \sim\left\{\begin{aligned}
\dot{x}_{a 2} & =A_{22} x_{a 2}-B_{22} \operatorname{Dz}\left(y_{c}\right) \\
\theta_{1} & =0 \\
\theta_{2} & =C_{2} x_{a 2}
\end{aligned}\right.
$$

This compensator is of order $n_{2}$ and that, apart from the nonlinear driving function, has linear dynamics. (8) looks very much like a reduced-order IMC compensator. The aforementioned reduced-order compensator is able to guarantee global exponential stability of the full nonlinear system.

Proposition 2. Consider the interconnection (1), (2) and (8). Let $r \equiv 0, d \equiv 0$ and $u_{m}=\operatorname{sat}\left(y_{c}\right)$. Let Assumption 1 and 2 be satisfied. Then the origin of the interconnected system is globally exponentially stable.

Proof: See appendix, Section 8.2.

Remark 2. It is emphasized that the reduced order compensator (8) is entirely linear. Note also that the compensator is not equivalent to a compensator calculated from the Jacobian linearisation of the model, but it is obtained by simply setting the nonlinear terms to zero. 


\subsection{A sub-optimal anti-windup design}

In section 3.2, it was proved that, despite omitting the $x_{a 1}$ dynamics, the system is globally exponentially stable. This compensator could be interpreted as a reduced-order IMC compensator because of its structure. However, IMC compensators of this type are well known to suffer problems with their transient performance [31], despite their global stabilising guarantees.

In common with techniques for linear systems (e.g. [5,32]), an extra "state-feedback-like" term is added to the $x_{a 2}$ dynamics of the AW compensator of Eq. (8), equal to $B_{22} F x_{a 2}$, where $F \in$ $\mathbb{R}^{m \times n}$. This term provides design freedom to the anti-windup problem and, as will be shown below leads to an LMI-based condition for compensator design. In this case, the improved anti-windup compensator state-space description becomes

$$
\mathcal{A W}_{I} \sim\left\{\begin{aligned}
\dot{x}_{a 2} & =A_{22} x_{a 2}-B_{22} \mathrm{Dz}\left(y_{c}\right)+B_{22} F x_{a 2} \\
\theta_{1} & =F x_{a 2} \\
\theta_{2} & =C_{2} x_{a 2}
\end{aligned}\right.
$$

Again, this compensator is of order $n_{2}$ and, apart from the input nonlinearity, has linear dynamics. The following Proposition shows that the improved compensator is able to guarantee global exponential stability of the full nonlinear system providing a certain LMI is satisfied.

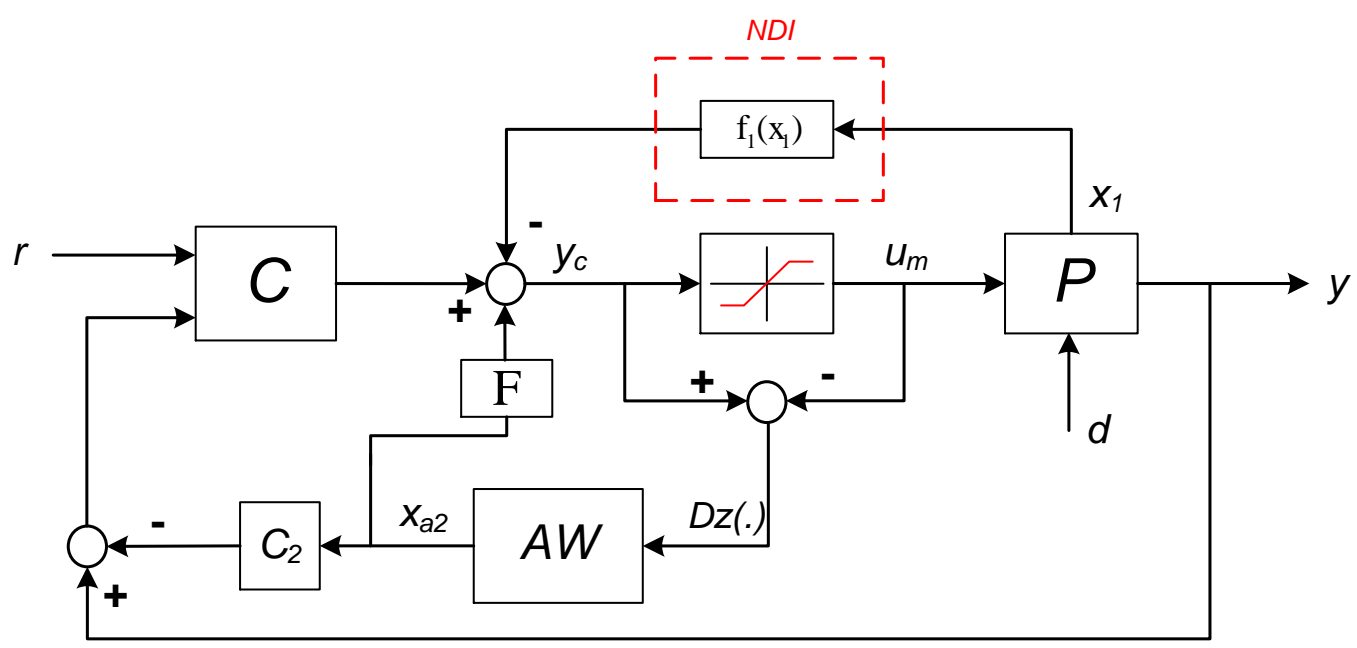

Fig. 3: System block diagram of the proposed reduced-order IMC-type anti-windup compensator

Proposition 3. Consider the interconnection of (1), (2) and (9). Let $r \equiv 0, d \equiv 0$ and $u_{m}=$ sat $\left(y_{c}\right)$. Let Assumption 1 and 2 be satisfied. Then there exists an anti-windup compensator of the form (9) which ensures the origin of the interconnected system is globally exponentially stable, provided there exist a symmetric positive definite matrix $\tilde{Q}>0$, a diagonal matrix $\tilde{U}>0$ and a matrix $\tilde{L}$ such that the following LMI is satisfied.

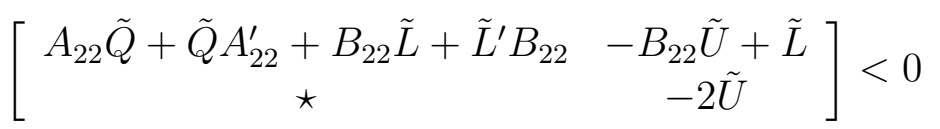


Furthermore, such an anti-windup compensator can be constructed as given in equation (9) with $F=\tilde{L} \tilde{Q}^{-1}$.

Proof: See appendix, Section 8.3.

Remark 3. Note that the improved AW compensator is, again, entirely linear and that it can be synthesised by simply solving an LMI problem. This is in contrast to the technique proposed in [24] where a matrix partial differential inequality needed to be solved. Note also, that when $F \equiv 0$ (and thus $L \equiv 0$ ), satisfaction of the LMI in Proposition 3 is guaranteed and hence the reduced order IMC compensator appears as a special case of Proposition 3, as one might expect.

The LMI (10) which appears in Proposition 3 could be combined with other approaches. One simple manner to adapt it would be to solve the LMI associated with linear anti-windup design appearing in [32] but for the reduced-order dynamics associated with the compensator (9). We term this approach the $\mathcal{L}_{2}$ approach since the linear LMIs deal with minimising a given $\mathcal{L}_{2}$ gain.

An alternative construction would replace the LMI (10) with the generalised eigenvalue problem (GEVP) involving the positive scalar $\lambda$

$$
\left[\begin{array}{cc}
A_{22} \tilde{Q}+\tilde{Q} A_{22}^{\prime}+B_{22} \tilde{L}+\tilde{L}^{\prime} B_{22}+\lambda \tilde{Q} & -B_{22} \tilde{U}+\tilde{L} \\
\star & -2 \tilde{U}
\end{array}\right]<0
$$

Maximising $\lambda$ then leads to a compensator with a maximum decay rate, which might lead to improved performance. We term this approach the decay rate approach. Note that the matrix inequality (11) can be solved using the GEVP function in the Matlab Robust Control Toolbox [33].

Remark 4. This paper focuses on global results and so the deadzone nonlinearity is captured by the Sector $[0, I]$. This may lead to anti-windup compensators which, for small levels and/or periods of saturation, lead to conservative performance. In order to improve small-signal performance, it is possible to use local sector bounds on the deadzone, of the sort proposed in [1] for example. This would enable one to compute greater (local) decay rates for systems which may lead to performance improvement. The price one pays for this is that the results hold only locally and global stability guarantees cannot be given.

\subsection{Input-to-state stability}

Propositions 1 - 3 give conditions which guarantee global asymptotic stability of the system in equations (1) and (2) when various different anti-windup compensators are used to assist the baseline controller. These propositions can all be extended to prove input-to-state stability (ISS) of the closed-loop interconnections in a fairly straightforward manner. The outline of these extensions is given here, although the details are left for the reader to confirm. In the case of the full-order nonlinear IMC, note that equation (6) takes the form

$$
\left\{\begin{aligned}
\dot{\xi} & =h(\xi)+\hat{B}_{1} \mathrm{Dz}\left(y_{c}\right)+\hat{B}_{2} w \\
y_{c} & =g(\xi)+\hat{D} w
\end{aligned}\right.
$$

where $\xi$ represents the concatenated state-vector of the system as mentioned in Appendix 8.1. The state equation is not necessarily Lipschitz, so the ISS results in Chapter 5 of [2] cannot be applied directly. However, using the same block-diagonal quadratic Lyapunov function as used in Appendix 8.1, $V(\xi)=\xi^{\prime} P \xi$ where $P=\operatorname{blockdiag}\left(P_{a}, P_{1}, P_{2}\right)$ and grouping the exogenous inputs into the vector $w=\left[\begin{array}{ll}r^{\prime} & d^{\prime}\end{array}\right]^{\prime}$, it can be shown that there are positive constants $\eta_{1}$ and $\eta_{2}$ such that

$$
\dot{V}(\xi) \leq-\eta_{1}\|\xi\|^{2}+\eta_{2}\|\xi\|\|w\|
$$


Using similar arguments to those found in Sections 5.2 and 5.3 of [2], it can then be proven that there exist further positive scalars, $\eta_{3}$ and $\eta_{4}$, such that

$$
\|\xi\| \leq \exp \left(-\eta_{3} t\right)\|\xi(0)\|+\eta_{4} \sup _{t \geq 0}\|w(t)\|
$$

which then implies that the system in (6) is ISS and that the origin is ultimately bounded. The same approach can be used to extend Propositions 2 and 3 to include ISS results. In all cases, once global stability has been established (as proved in the appendices), ISS can be proved relatively easily by noting that the exogenous inputs enter the plant and the controller state-equations, (1) and (2) respectively, linearly.

\section{Application to a Wave Energy Converter (WEC)}

The inspiration for the development of the proposed class of AW compensators was a saturation problem reported in the control of WEC [28]. WECs have received considerable attention over recent years due to their ability to supply sustainable energy and potable water. The control of such devices is interesting and challenging and in [34,35] some advanced predictive control strategies have been proposed and compared with simpler traditional techniques. The attractive feature of these predictive techniques is that control signal amplitude limits are accounted for in the underlying finite horizon optimisation problem which determines the controller. Unfortunately, the simpler linear techniques which are often preferred do not account for these constraints a priori and then may require anti-windup compensation in order to prevent performance loss which may occur during extreme weather conditions.

This section reports the application of the anti-windup techniques developed in the previous sections to a WEC model developed in $[27,28]$. This model describes the dynamics of a potable water production process consisting of the mechanical wave energy converter dynamics and the dynamics of the desalination process. In [27,28] a nonlinear proportional integral (PI) controller, of exactly the form considered in this paper was applied to the problem and found to provide satisfactory performance if saturation was not encountered. Once the saturation limits were applied, performance became unsatisfactory [27].

The WEC studied here consists of a point-absorber oscillating in heave. The point-absorber is coupled to a Power Take-Off unit (PTO), which converts wave energy into pressure. The aim of the controller is to regulate the pressure on the WEC's Reverse Osmosis (RO) membrane to $6 \cdot 10^{6}$ $\mathrm{Pa}$ in order to ensure an efficient desalination process. To prevent poor quality water production or damage to the RO membrane, the deviation from the $6 \cdot 10^{6}$ Pa reference should be less than $3 \%$.

\subsection{State-space model}

The model of the WEC, around a desired equilibrium pressure of $\overline{P_{\text {ro }}}=6 \cdot 10^{6} \mathrm{~Pa}$, can be represented by equation (1) where the state-space matrices are: 


$$
\begin{aligned}
A_{11} & =\left[\begin{array}{cc}
0 & 1 \\
-\frac{S}{M} & -\frac{R_{f}+R_{c}}{M}
\end{array}\right] \quad A_{12}\left(x_{1}\right)=\left[\begin{array}{c}
0 \\
-\frac{\mathbf{A}_{\mathbf{p}}^{*}}{M}
\end{array}\right] \\
A_{21}\left(x_{1}\right) & =\left[\begin{array}{cc}
0 & \frac{\mathbf{A}_{\mathbf{p}}^{*}}{C}
\end{array}\right] x_{1} \quad A_{22}=-\frac{\left(\overline{P_{r o}}-P_{o s m}\right) p_{r o}}{C \cdot P_{r o}} \\
B_{11} & =\left[\begin{array}{c}
0 \\
\frac{1}{M}
\end{array}\right] \quad B_{21}=0 \quad B_{22}=\frac{-C_{r} \sqrt{\overline{P_{r o}}}}{200 C}
\end{aligned}
$$

A detailed explanation of the model, and numerical values for the parameters, can be found in [27]. The states are the WEC heave position $x_{11}$, heave velocity $x_{12}$ and the PTO state, $x_{2}$. The model in Eq. (15) is nonlinear, since the term $\mathbf{A}_{\mathbf{p}}^{*}$ switches according to the sign of $x_{12}$, where $x_{1}$ has been partitioned as $x_{1}=\left[\begin{array}{ll}x_{11} & x_{12}\end{array}\right]$; that is

$$
\mathbf{A}_{\mathbf{p}}^{*}=\operatorname{sign}\left(x_{12}\right) A_{p}= \begin{cases}A_{p}, & \text { if } x_{12}>0 \\ 0, & \text { if } x_{12}=0 \\ -A_{p}, & \text { if } x_{12}<0\end{cases}
$$

where $A_{p}$ is the pump area [28]. $\mathbf{A}_{\mathbf{p}}^{*}$ is the only source of nonlinearity and is such that Items 3 and 4 of Assumption 1 are satisfied. The first two requirements of Assumption 1 are also satisfied because the matrices $A_{11}$ and $A_{22}$ are both Hurwitz.

\subsection{Excitation force}

The excitation force model represents the motion of the ocean waves exerted on the buoy causing it to oscillate. The excitation force is an exogenous input (acting as a disturbance) to the system and is represented by a model producing irregular waves based on the Pierson-Moskowitz (PM) [36] energy spectrum for fully developed seas. The PM spectrum is representative of a large number of ocean locations and is given by

$$
E_{\omega}(\omega)=\frac{0.11 H_{\frac{1}{3}}^{2} T_{1}}{2 \pi}\left(\frac{\omega T_{1}}{2 \pi}\right)^{-5} \exp \left[-0.44\left(\frac{\omega T_{1}}{2 \pi}\right)^{-4}\right]
$$

where $H_{\frac{1}{3}}$ is the significant wave height, $T_{1}$ is the mean wave period and $\omega$ is the wave frequency.

The time series corresponding to the spectrum of Fig. 4 can be calculated by dividing it into 100 equally spaced frequencies at intervals of $\Delta \omega=0.0325 \mathrm{rad} / \mathrm{s}$ up to a maximum frequency of $3.2459 \mathrm{rad} / \mathrm{s}$ [28]. The surface elevation is then calculated as

$$
\eta(t)=\sum_{i=1}^{100} \kappa(i) \sin (\omega(i) t+\psi(i))
$$

where

$$
\kappa(i)=\sqrt{2 E_{\omega}(\omega(i)) \Delta \omega}
$$

and $\psi(i)$ are random phase angles distributed uniformly from 0 to $2 \pi$. The excitation force corresponding to the energy spectrum of Fig. 4 gives rise to the excitation force, $F_{e}$, depicted in Fig. 5 [see [28] for details]. 


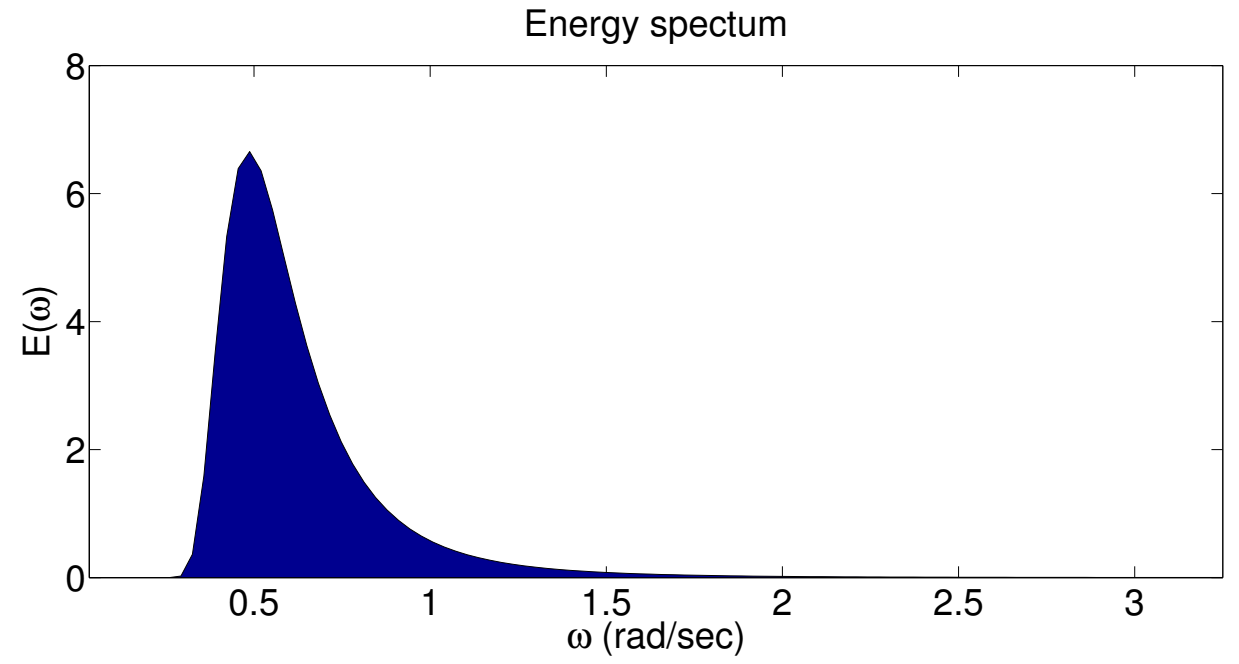

Fig. 4: Energy spectrum characterised by $H_{1 / 3}=6 \mathrm{~m}$ and $T_{1}=10 \mathrm{sec}$.

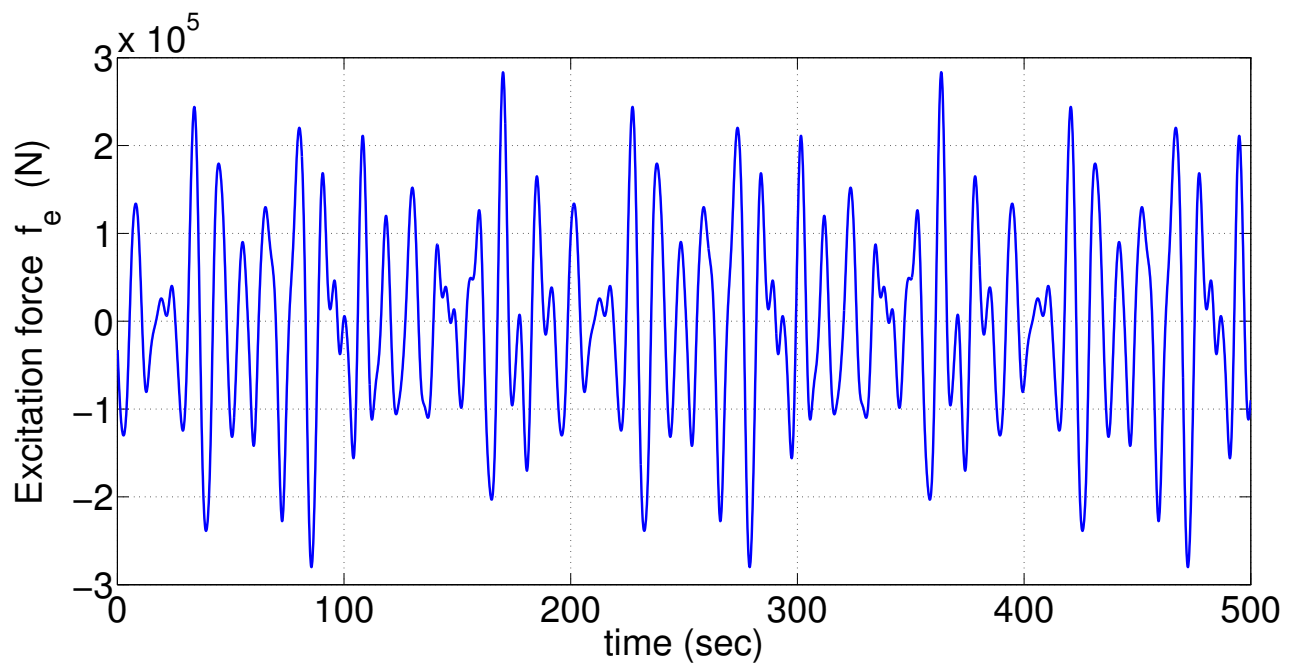

Fig. 5: Realisation of the excitation force corresponding to the energy spectrum of Fig. 4. 


\subsection{Nominal controller}

References [27, 28] suggested a modified PI controller, featuring partial feedback-linearisation. When no saturation occurs, this controller decouples the PTO subsystem from the mechanical subsystem and allows excellent pressure regulation to be achieved while also guaranteeing global asymptotic stability [30]. The controller dynamics have the form given in equation (2), viz:

$$
\mathcal{C}_{w e c} \sim\left\{\begin{aligned}
\dot{x}_{c} & =\underbrace{0}_{A_{c}} x_{c} \underbrace{-1}_{B_{c y}} \underbrace{x_{2}}_{y}+\underbrace{1}_{B_{c r}} r \\
u & =\underbrace{K_{i}}_{C_{c}} x_{c} \underbrace{K_{2}}_{D_{c y} K_{p}}+\underbrace{\left(K_{p}+K_{f f}\right)}_{y} r+\underbrace{K_{s}(x)}_{D_{c r}}
\end{aligned}\right.
$$

where the PI gains $K_{p}=\frac{-\left(\mu_{1}+\mu_{2}\right)+A_{22}}{B_{22}}$ and $K_{i}=\frac{\mu_{1} \mu_{2}}{B_{22}}$, and $\mu_{1}=\mu_{2}=0.12$ determine the closedloop pole locations $[27,28]$. The feedforward controller gain $K_{f f}=-\frac{A_{22}}{B_{22}}$ and $K_{s}(x)$ is a nonlinear term chosen to cancel the nonlinear terms associated with the PTO state equation,

$$
K_{s}(x)=-\frac{1}{B_{22}} \frac{A_{p}}{C} \operatorname{sign}\left(x_{12}\right) x_{12}=-\frac{1}{B_{22}} \frac{A_{p}}{C}\left|x_{12}\right|
$$

The magnitude of the control signal is limited between $0-100 \%{ }^{1}$. The non-symmetric saturation function used here is defined as

$$
\operatorname{sat}(u)= \begin{cases}\bar{u}, & u \geq \bar{u} \\ u, & \underline{u}<u<\bar{u} \\ \underline{u}, & u \leq \underline{u}\end{cases}
$$

where $\underline{u}=0$ and $\bar{u}=100$. Reference [28] identifies actuator saturation as a regular occurrence which impedes the satisfactory functioning of this type of WEC, with performance degrading dramatically when saturation occurs. Noting the merits of the nominal controller, AW appears a logical mechanism for addressing these saturation problems.

\section{Simulation results}

The performance of several anti-windup compensators is compared in this section:

1. A standard linear IMC compensator. This compensator has been designed according to equation (16) in [32] with $F \equiv 0$ and the state-space realisation of the linearised plant taken from equation (15) with $\mathbf{A}_{\mathbf{p}}{ }^{*}=A_{p}$. This implies the anti-windup compensator has been designed based only on linearised dynamics of the plant, not the full nonlinear equations as with compensators (6) and (8) above.

2. The nonlinear IMC AW compensator (6) from Proposition 1.

3. The reduced-order IMC AW compensator (8) from Proposition 2. Although this compensator has been designed taking the nonlinear dynamics of the plant into account, the dynamics of the compensator itself are purely linear

\footnotetext{
${ }^{1}$ The control signal corresponds to the position of a throttle valve, which ranges from fully closed to fully open, hence $0-100 \%$.
} 
4. An improved reduced-order compensator (9) from Proposition 3 and using the LMI from equation (23) in [32] to enforce the inequality (10). If the plant were linear, this would correspond to the minimisation of a useful $\mathcal{L}_{2}$ performance map. Of course, here the plant dynamics are nonlinear so there is no guarantee that such an $\mathcal{L}_{2}$ performance measure is minimised, but the approach appears to be useful.

5. An improved reduced-order compensator (9) from Proposition 3 but with inequality (11) used to enforce inequality (10) and to maximise the decay rate of the anti-windup compensator state.

Table 1 compares the responses of the system under various different wave profiles, with $r(t)$ fixed as a ramp-type function with a steady-state value of $6 \cdot 10^{6} P_{a}$, the pressure required for the RO process. Illustrative time-histories are given in Figures 6-8. Figure 6 shows the WEC pressure time histories for three cases: without saturation; with saturation but no anti-windup; and with saturation and linear IMC anti-windup. Note that when saturation is present, the system exhibits overshoot and a long settling time, but when the linear IMC anti-windup compensator is added, little improvement is observed and tracking performance is lost. Figure 7 shows a similar set of responses, but this time responses of the IMC-like compensators of Propositions 1 and 2 are included. Despite being able to offer stability, these compensators again lead to poor performance and again tracking is lost. Figure 8 shows the pressure time-histories of the two compensator produced from Proposition 3: in this case it is noted that both compensators lead to improved performance with tracking restored, and settling time and overshoot reduced.

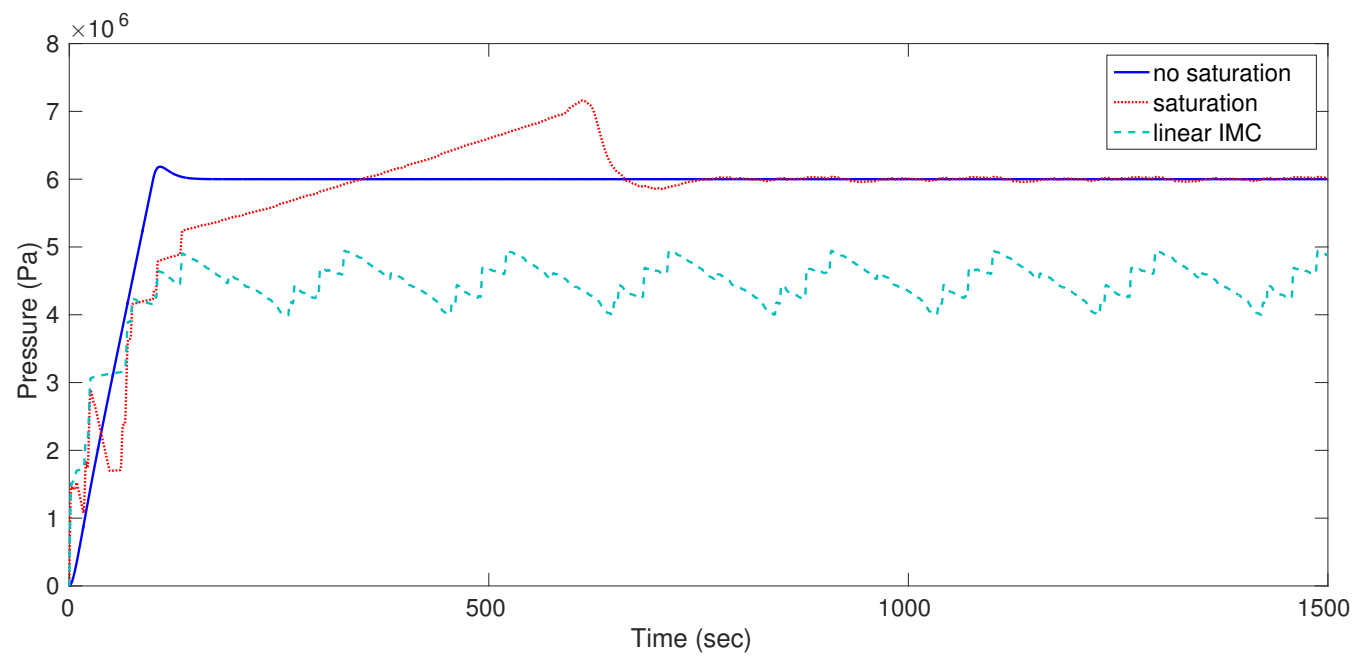

Fig. 6: Simulation for $H=1.1 m, T_{1}=10 \mathrm{sec}$ - comparison between no AW, saturation and linear IMC compensator.

In Fig. 9 one can see the control signals of the saturated closed-loop system, the linear IMC and the proposed AW schemes. The unconstrained control signal has been omitted for readability purposes, as its magnitude reached a value of 2500 obstructing the rest of the signals. The linear IMC as well as the nonlinear AW schemes of Propositions $1 \& 2$ exhibit identical control signals. The decay rate AW scheme of Proposition 3 has the drawback of a highly switching control signal, which would make its implementation quite challenging in practice. On the other hand, the reduced-order AW compensator designed using method appearing in [32] ( $L_{2} \mathrm{AW}$ scheme of 


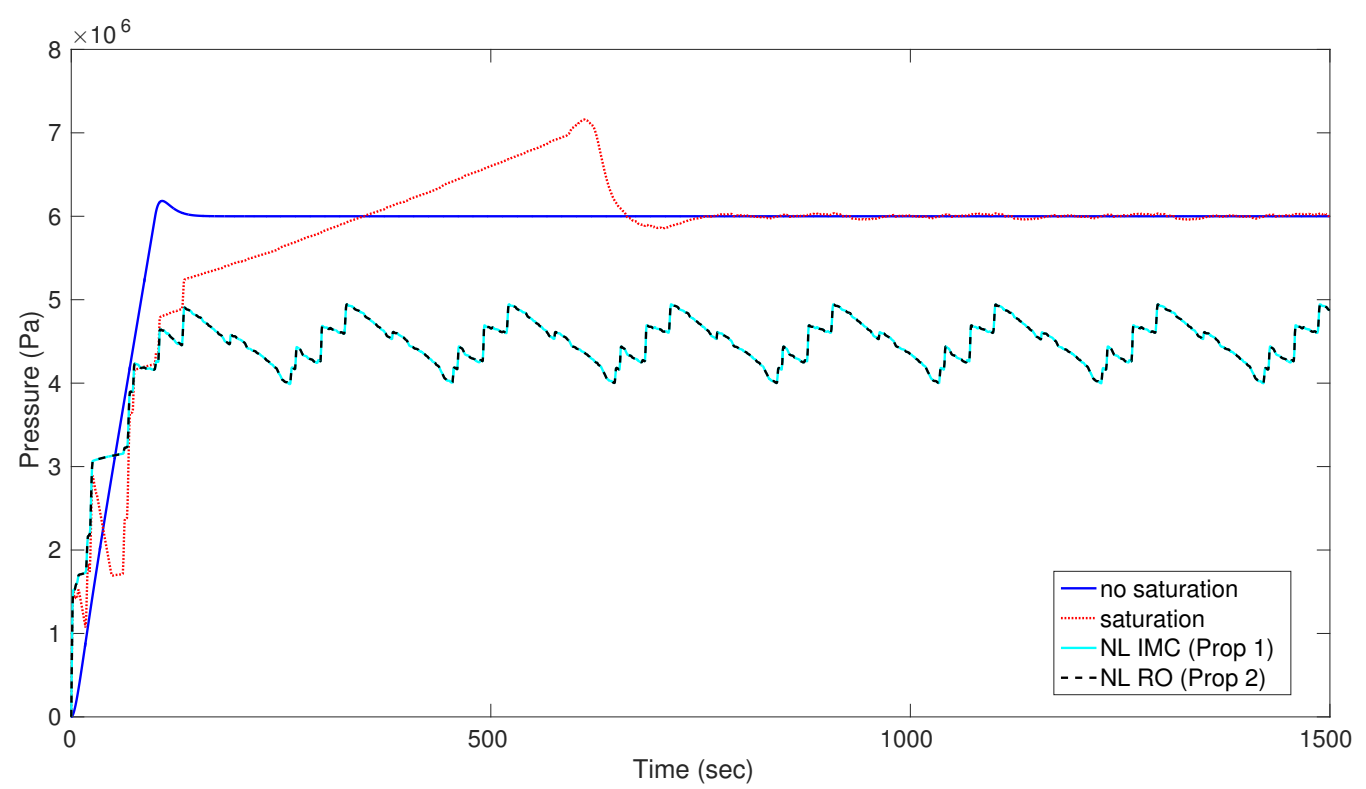

Fig. 7: Simulation for $H=1.1 m, T_{1}=10 \mathrm{sec}$ - comparison between no AW, saturation and AW compensators of Prop. $1 \& 2$.

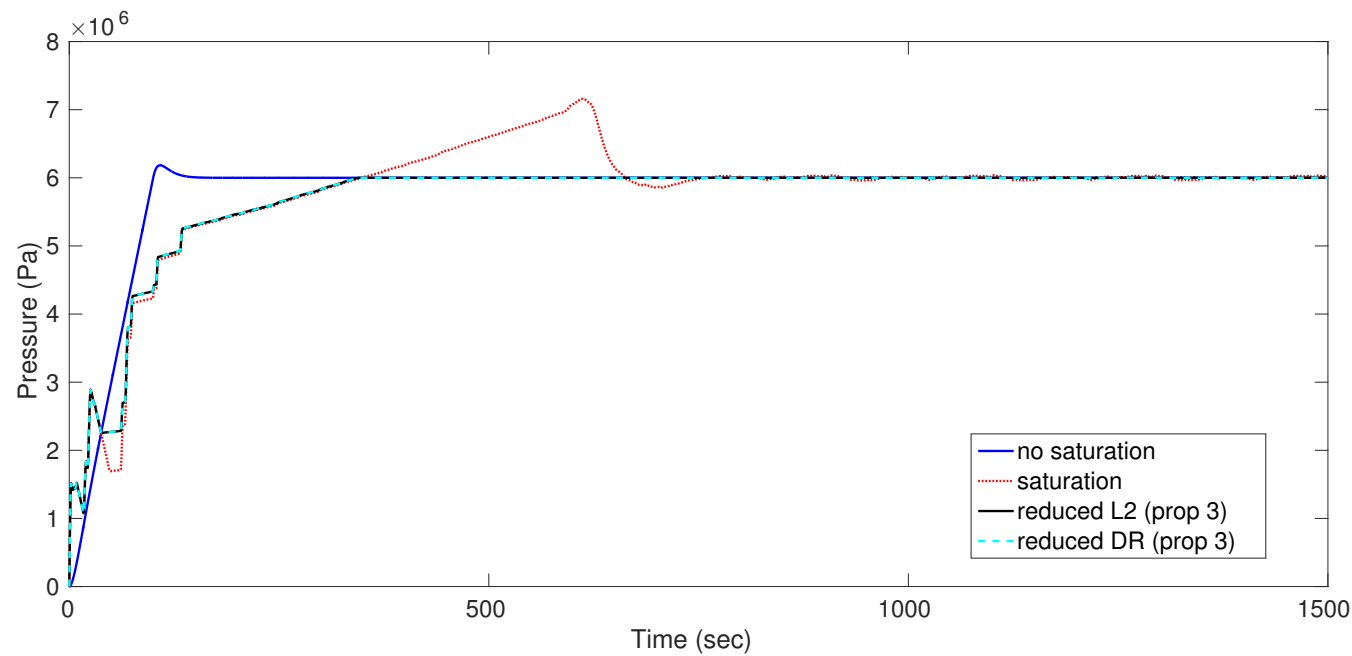

Fig. 8: Simulation for $H=1.1 m, T_{1}=10 \mathrm{sec}$ - comparison between no AW, saturation and AW compensators from Prop. 3. 
Proposition 3) exhibits a control signal which contains much lower activity compared to the decay rate maximiser one.
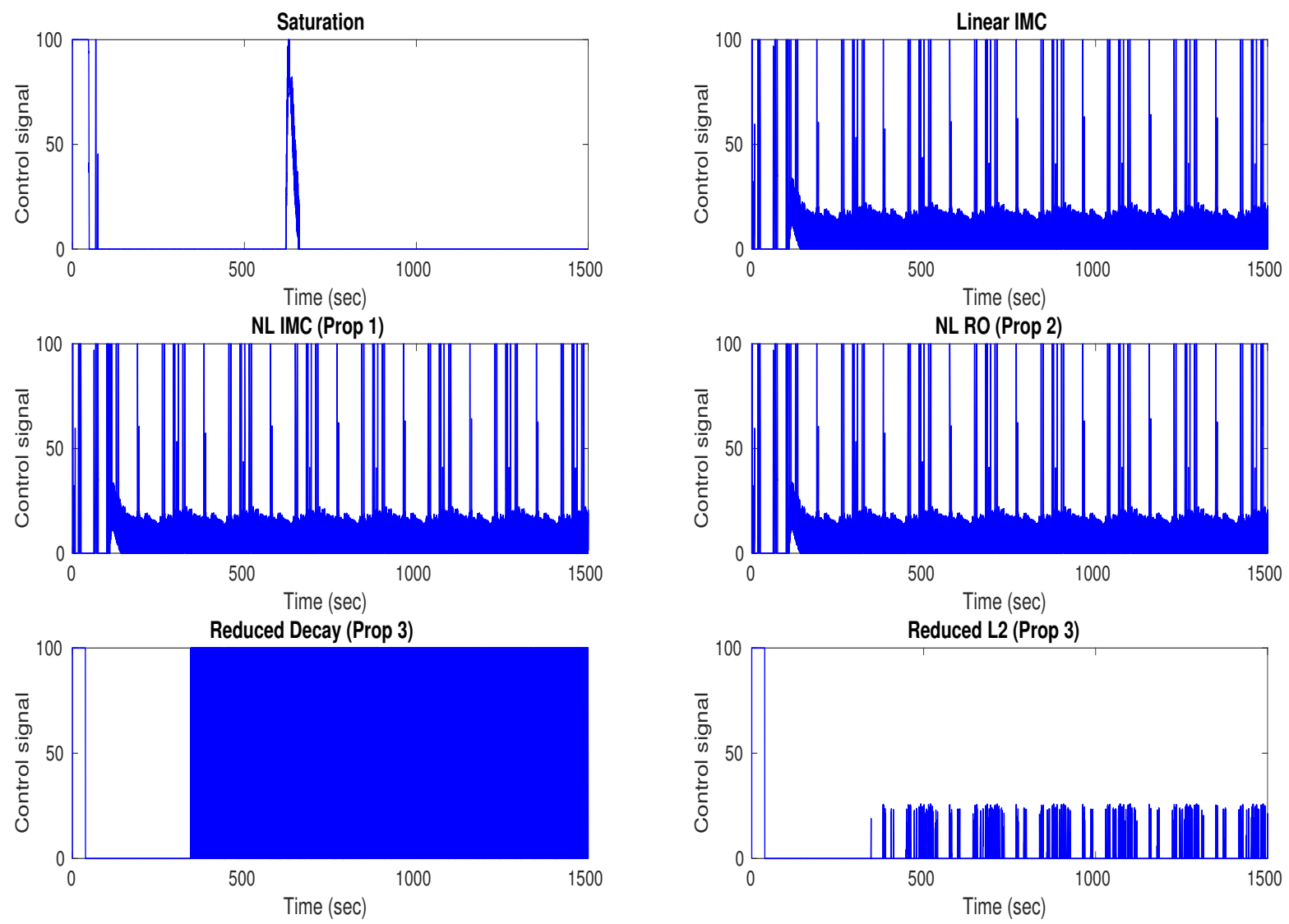

Fig. 9: Comparison of the constrained control signals.

\section{Conclusions}

Three IMC-type compensators which guarantee global exponential stability have been proposed for a class of input-constrained partially linearisable nonlinear systems. The first is a natural counterpart to the IMC compensator derived in [24]; the second is a reduced-order IMC compensator which only features linear dynamics, giving it twofold practical appeal. The third one is of similar structure to the second one, however it contains an extra term that can be used to maximise the decay rate, hence improving performance.

The results derived here have been applied to, and indeed were motivated by, a WEC pressure regulation case study. Results from this case study have indicated that the new nonlinear IMC and linear reduced-order AW compensators stabilise the system, however they are unable to track the reference. In contrast, the new linear AW compensator with decay rate maximiser performed well although the window in which tracking was typically achieved (waves of height $1-1.3 \mathrm{~m}$ ) is quite narrow. 


\section{References}

[1] Tarbouriech S., Turner M.C.: Anti-windup design: an overview of some recent advances and some open problems, IET Control Theory and Applications, 2009, 3, (1), pp. 1-19

[2] Galeani S., Tarbouriech S., Turner M.C., Zaccarian L.: A Tutorial on modern anti-windup design, Europ. Journ. of Control, 2009, 15, (3-4), pp. 418-440

[3] Glattfelder A.H., Schaufelberger W.: Control systems with input and output constraints, (Springer-Verlag, London, 2003)

[4] Hippe P.: Windup in control. Its effects and their prevention, (AIC, Springer, Germany, 2006)

[5] Zaccarian L., Teel A.R.: Modern Anti-windup Synthesis: Control Augmentation for Actuator Saturation, (Princeton University Press, New Jersey, 2011)

[6] Tarbouriech S., Garcia G., Gomes da Silva Jr. J.M., Queinnec I.: Stability and Stabilization of Linear Systems with Saturating Actuators, (Springer Verlag, 2011)

[7] Mulder E.F., Kothare M.V., Morari M.: Multivariable anti-windup controller synthesis using linear matrix inequalities, Automatica, 2001, 37, pp. 1407-1416

[8] Grimm G., Hatfield J., Postlethwaite I., Teel A.R., Turner M.C., Zaccarian L.: Anti-windup for stable linear systems with input saturation: an LMI based synthesis, IEEE Trans. on Autom. Control, 2003, 48, 9, pp. 1509-1525

[9] Saeki M., Wada N.: Synthesis of a static anti-windup compensator via linear matrix inequalities, Int. Journ. of Robust and Nonlinear Control, 2002, 12, 10, pp. 927-953

[10] Wu W., Jayasuriya S.: A New QFT Design Methodology for Feedback Systems Under Input Saturation, Journ. of Dynamical Systems, Measuring and Contol, 2001, 123, pp. 225-23

[11] Kerr M., Villota E., Jayasuriya S.: Robust Anti-windup Design for Input Constrained SISO Systems, 8th Int. Symp. on QFT and Robust Frequency Domain Methods, 2008

[12] Kerr M., Turner M.C., Villota E., Jayasuriya S., Postlethwaite I.: A robust anti-windup design procedure for SISO systems, Int. Journ. of Control, 2011, 84, 2, pp. 351-369

[13] Morabito F., Teel A.R., Zaccarian L.: Nonlinear Antiwindup Applied to Euler-Langrange Systems, IEEE Trans. on Robotics and Automation, 2004, 20, 3, pp. 526-537

[14] Teel A.R., Kapoor N.: The $\mathcal{L}_{2}$ Anti-windup Problem: Its Definition and Solution, Europ. Control Conference, 1997

[15] Kahveci N.E., Ioannou P.A., Mirmirani M.D.: Adaptive LQ Control With Anti-Windup Augmentation to Optimize UAV Performance in Autonomous Soaring Applications, IEEE Trans. on Control Systems Technology, 2008, 16, 4, pp. 691-707

[16] Sofrony Esmeral J., Turner M.C., Postlethwaite I.: Anti-windup using Riccati equations for systems with rate-limits, Int. Journ. of Control, 2010, 83, 2, pp. 233-245

[17] Coutinho D.F., Bazanella A.S., Trofino A., e Silva A.S.: Stability analysis and control of a class of differential-algebraic nonlinear systems, Int. Journ. of Robust and Nonlinear Control, 2004, 14, 16, pp. 1301-1326 
[18] Oliveira M.Z., da Silva Jr. J.M.G., Coutinho D.F., Tarbouriech S.: Anti-windup Design for a Class of Nonlinear Control Systems, IFAC World Congress, Milan, 2011

[19] da Silva Jr. J.M.G., Corso J., Castelan E.B.: Output feedback stabilization for systems presenting sector-bounded nonlinearities and saturating inputs, IFAC World Congress, Seoul, 2008

[20] da Silva Jr. J.M.G., Turner M.C.: Static anti-windup for systems with sector-bounded nonlinearities, Amer. Control Conference, Montreal, 2012

[21] Doyle III F.J.: An anti-windup input-output linearisation scheme for SISO systems, Journal of Process Control, 1999, 9, 3, pp. 213-220

[22] Kapoor N., Daoutidis P.: An observer based anti-windup scheme for nonlinear systems with input constraints, Int. Journ. of Control, 1999, 72, 1, pp. 18-29

[23] Herrmann G., Turner M.C., Menon P.P., Bates D., Postlethwaite I.: Anti-windup synthesis for nonlinear dynamic inversion controllers, IFAC Robust Controller Design Symposium (ROCOND), Toulouse, 2006

[24] Herrmann G., Menon P.P., Turner M.C., Bates D., Postlethwaite I.: Anti-windup synthesis for nonlinear dynamic inversion control schemes, Int. Journ. of Robust and Nonlinear Control, 2010, 20, 13, pp. 1465-1482

[25] Valmorbida G., Tarbouriech S., Turner M.C., Garcia G.: Anti-windup for NDI quadratic systems, Nonlinear Control Design Symposium (NOLCOS), Milan, 2011

[26] Weston P.F., Postlethwaite I.: Linear Conditioning for systems containing saturating actuators, Automatica, 2000, 36, 9, pp. 1347-1354

[27] Nolan G.A., Ringwood J.V.: Control of a Heaving Buoy Wave Energy Converter for Potable Water Production, Irish Signal and Systems Conf., 2006, Dublin

[28] Nolan G.A.: 'Modelling and Optimisation of a Heaving Buoy Wave Energy Converter for Potable Water Production'. PhD thesis, National University of Ireland, Maynooth, 2006

[29] Lekka A., Turner M.C., Menon P.P.: Full and reduced order IMC anti-windup compensators for a class of nonlinear systems with application to wave energy converter control, Amer. Control Conference, 2013, Washington DC

[30] Lekka A., Turner M.C., Ringwood J.V.: A class of globally stabilising controllers for the control of wave energy devices for potable water production, IEEE Int. Conf. on Control Applications, 2012, Dubrovnik

[31] Grimm G., Postlethwaite I.,Teel A.R., Turner M.C., Zaccarian L.: Case studies using linear matrix inequalities for optimal anti-windup synthesis, Europ. Journ. of Control, 2003, 9, 5, pp. 463-473

[32] Turner M.C., Herrmann G., Postlethwaite I.: Incorporating Robustness Requirements Into Antiwindup Design, IEEE Trans. on Automatic Control, 2007, 52, 10, pp. 1842-1855

[33] Balas G., Chiang R., Packard A., Safonov M.: 'Robust Control Toolbox 3 - User's Guide' 
[34] Li G., Weiss G., Mueller M., Townley S., Belmont M.R.: Wave energy converter control by wave prediction and dynamic programming, Renewable Energy, 2012, 48, 0, pp. 392-403

[35] Abraham E., Kerrigan E.: Optimal active control of a wave energy converter, IEEE 51st Annual Conf. on Decision and Control, 2012, Grand Wailea, Maui, Hawaii

[36] Pierson Jr. W.J., Moskowitz L.: A Proposed Spectral Form for Fully Developed Wind Seas Based on the Similarity Theory of S. A. Kitaigorodskii, Journal of Geophysical Research, 1964, 69, 24, pp. 5181-5190

[1] Da Silva J.M.G, Tarbouriech S.: Antiwindup design with guaranteed regions of stability: an LMI-based approach, IEEE Transactions on Automatic Control, 2005, 1, 50, pp. 106-111

[2] Khalil, H.K.: 'Nonlinear Systems' (Prentice Hall, London, 3rd edn. 2002)

\section{Appendix}

\section{Proofs}

\subsection{Stability proof for the nonlinear IMC AW compensator of Section 3.1}

Consider the closed-loop interconnection represented by equation (7). A composite Lyapunov function approach is adopted where each individual Lyapunov function corresponds to a "subsystem". It is then shown that the time derivative of this composite Lyapunov function is negative definite.

8.1.1. Lyapunov function candidate $V_{a}\left(x_{a}\right)$ : Let $V_{a}\left(x_{a}\right)=\alpha x_{a}^{\prime} P_{a} x_{a}$, with $P_{a}>0$.

The time derivative of $V\left(x_{a}\right)$ along the trajectories of the $x_{a}$ subsystem (6) is

$$
\begin{aligned}
\dot{V}_{a}\left(x_{a}\right) & =2 \alpha x_{a}^{\prime} P_{a}\left[\begin{array}{c}
A_{11}\left(x_{a 1}\right)+A_{12}\left(x_{a 1}\right) x_{a 2} \\
A_{21}\left(x_{a 1}\right)+A_{22} x_{a 2}
\end{array}\right] \\
& -2 \alpha x_{a}^{\prime} P_{a}\left[\begin{array}{ll}
0 & B_{22}^{\prime}
\end{array}\right]^{\prime} \phi
\end{aligned}
$$

where $\phi:=\mathrm{Dz}\left(y_{c}\right)$. Regarding the $x_{a}$ subsystem, it is split in an "unforced" part and in a "forced" part (due to the deadzone). Item 1 of Assumption 1 implies there exists an $\epsilon>0$ such that

$$
\dot{V}_{a}\left(x_{a}\right) \leq-\alpha \epsilon\left\|x_{a}\right\|^{2}-2 \alpha x_{a}^{\prime} P_{a}\left[\begin{array}{ll}
0 & B_{22}^{\prime}
\end{array}\right]^{\prime} \phi
$$

Then using Fact 1 we have

$$
\begin{aligned}
\dot{V}_{a}\left(x_{a}\right) & \leq-\alpha \epsilon\left\|x_{a}\right\|^{2}-2 \alpha x_{a}^{\prime} P_{a}\left[\begin{array}{ll}
0 & B_{22}^{\prime}
\end{array}\right]^{\prime} \phi \\
& +2 \phi^{\prime} W[\underbrace{C_{2 c} e_{2 c}-f_{1}\left(x_{1}\right)+f_{1}\left(x_{a 1}\right)}_{y_{c} \text { from Eq. } 7}-\phi] \\
& \leq\left[\begin{array}{c}
x_{a} \\
\phi
\end{array}\right]^{\prime}\left[\begin{array}{cc}
-\alpha \epsilon I_{n} & \alpha P_{a}\left[\begin{array}{c}
0 \\
B_{22}
\end{array}\right] \\
\star & -2 W
\end{array}\right]\left[\begin{array}{c}
x_{a} \\
\phi
\end{array}\right] \\
& +2\|\phi\|\|W\|\left(\left\|C_{2 c} e_{2 c}\right\|+\left\|f_{1}\left(x_{1}\right)-f_{1}\left(x_{a 1}\right)\right\|\right)
\end{aligned}
$$



( [2])

where $W$ is some positive definite diagonal matrix to be decided. Using Young's inequality

$$
2 x^{\prime} y \leq \gamma\|x\|^{2}+\frac{1}{\gamma}\|y\|^{2} \quad \forall x \in \mathbb{R}^{p}, y \in \mathbb{R}^{q}, \gamma>0
$$

it follows, for any $\delta_{a}>0$, that

$$
\begin{aligned}
\dot{V}\left(x_{a}\right) & \leq\left[\begin{array}{c}
x_{a} \\
\phi
\end{array}\right]^{\prime}\left[\begin{array}{cc}
-\alpha \epsilon I_{n} & \left.\alpha P_{a}\left[\begin{array}{c}
0 \\
B_{22}
\end{array}\right]\right]\left[\begin{array}{c}
x_{a} \\
\phi
\end{array}\right] \\
\star & -2 W
\end{array}\right] \\
& +\delta_{a}\|\phi\|^{2}\|W\|^{2}+\frac{2}{\delta_{a}}\left(\left\|C_{2 c}\right\|^{2}\left\|e_{2 c}\right\|^{2}+k_{1}^{2}\left\|e_{1}\right\|^{2}\right)
\end{aligned}
$$

8.1.2. Lyapunov function candidate $V_{1}\left(e_{1}\right)$ : Let $V_{1}\left(e_{1}\right)=e_{1}^{\prime} P_{1} e_{1}$ with $P_{1}>0$. The time derivative along the trajectories of the $e_{1}$ sub-system is then

$$
\begin{aligned}
\dot{V}_{1}\left(e_{1}\right) & =2 e_{1}^{\prime} P_{1}\left[A_{11} e_{1}+A_{12}\left(x_{1}\right) x_{2}-A_{12}\left(x_{a 1}\right) x_{a 2}\right] \\
& \leq 2 e_{1}^{\prime} P_{1} A_{11} e_{1}+2 e_{1}^{\prime} P_{1}\left[A_{12}\left(x_{1}\right) x_{2}-A_{12}\left(x_{a 1}\right) x_{a 2}\right] \\
& \leq e_{1}^{\prime}\left(P_{1} A_{11}+A_{11}^{\prime} P_{1}\right) e_{1}+2\left\|e_{1}\right\|\left\|P_{1}\right\| \| A_{12}\left(x_{1}\right) x_{2} \\
& -A_{12}\left(x_{a 1}\right) x_{a 2} \|
\end{aligned}
$$

Adding and subtracting $A_{12}\left(x_{a 1}\right) x_{2}$ gives

$$
\begin{aligned}
& A_{12}\left(x_{1}\right) x_{2}-A_{12}\left(x_{a 1}\right) x_{a 2} \\
& =A_{12}\left(x_{1}\right) x_{2}-A_{12}\left(x_{a 1}\right) x_{2}+A_{12}\left(x_{a 1}\right) x_{2} \\
& -A_{12}\left(x_{a 1}\right) x_{a 2} \\
& =\left[A_{12}\left(x_{1}\right)-A_{12}\left(x_{a 1}\right)\right] x_{2}+A_{12}\left(x_{a 1}\right) e_{2}
\end{aligned}
$$

Using item 4 of Assumption 1, the norm can be bounded by the norms of the states $e_{2 c}$ and $x_{a}$

$$
\begin{aligned}
\| A_{12}\left(x_{1}\right) & x_{2}-A_{12}\left(x_{a 1}\right) x_{a 2} \| \\
& \leq\left\|A_{12}\left(x_{1}\right)-A_{12}\left(x_{a 1}\right)\right\|\left\|x_{2}\right\|+\left\|A_{12}\left(x_{a 1}\right)\right\|\left\|e_{2}\right\| \\
& \leq\left(\left\|A_{12}\left(x_{1}\right)\right\|+\left\|A_{12}\left(x_{a 1}\right)\right\|\right)\left\|x_{2}\right\|+\beta\left\|e_{2}\right\| \\
& \leq 2 \beta\left\|x_{2}\right\|+\beta\left\|e_{2}\right\| \\
& \leq 3 \beta\left\|e_{2}\right\|+2 \beta\left\|x_{a 2}\right\| \\
& \leq 3 \beta\left\|e_{2 c}\right\|+2 \beta\left\|x_{a}\right\|
\end{aligned}
$$

where the terms involving $\beta$ arise from repeated application of item 4 of Assumption 1. This implies, for any $\delta_{1}$, that $\dot{V}\left(x_{a}\right)$ in (31) can be bounded by

$$
\begin{aligned}
\dot{V}_{1}\left(e_{1}\right) & \leq e_{1}^{\prime}\left(P_{1} A_{11}+A_{11}^{\prime} P_{1}\right) e_{1} \\
& +2\left\|e_{1}\right\|\left\|P_{1}\right\| \beta\left(3\left\|e_{2 c}\right\|+2\left\|x_{a}\right\|\right) \\
& \leq e_{1}^{\prime}\left(P_{1} A_{11}+A_{11}^{\prime} P_{1}+\delta_{1}\left\|P_{1}\right\|^{2} I\right) e_{1} \\
& +\frac{2 \beta^{2}}{\delta_{1}}\left(9\left\|e_{2 c}\right\|^{2}+4\left\|x_{a}\right\|^{2}\right)
\end{aligned}
$$


8.1.3. Lyapunov function candidate $V_{2}\left(e_{2 c}\right)$ : Let $V_{2}\left(e_{2 c}\right)=e_{2 c}^{\prime} P_{2} e_{2 c}$ with $P_{2}>0$. The time derivative along the trajectories of the $e_{2 c}$ sub-system is

$$
\dot{V}_{2}\left(e_{2 c}\right)=e_{2 c}^{\prime}\left(A_{2 c}^{\prime} P_{2}+P_{2} A_{2 c}\right) e_{2 c}
$$

Sum of Lyapunov function candidates.The state-vector of the complete system can be written as $\xi:=\left[e_{1}^{\prime}, e_{2 c}^{\prime}, x_{a}\right]^{\prime}$, and the Lyapunov functions for the subsystems summed to get

$$
V_{t o t}(\xi):=V_{a}\left(x_{a}\right)+V_{1}\left(e_{1}\right)+V_{2}\left(e_{2 c}\right)>0 \quad \forall\left(e_{1}, e_{2 c}, x_{a}\right) \neq 0
$$

$V_{t o t}$ is positive definite and radially unbounded. From the bounds on $\dot{V}_{a}\left(x_{a}\right), \dot{V}_{1}\left(e_{1}\right)$ and $\dot{V}_{2}\left(e_{2 c}\right)$, its derivative is bounded by

$$
\begin{aligned}
\dot{V}_{\text {tot }}(\xi)= & \dot{V}_{a}\left(x_{a}\right)+\dot{V}_{1}\left(e_{1}\right)+\dot{V}_{2}\left(e_{2 c}\right) \\
\leq & {\left[\begin{array}{c}
x_{a} \\
\phi
\end{array}\right]^{\prime}\left[\begin{array}{cc}
\mathcal{E} & \mathcal{H} \\
\star & \mathcal{J}
\end{array}\right]\left[\begin{array}{c}
x_{a} \\
\phi
\end{array}\right] } \\
& +e_{1}^{\prime}\left(P_{1} A_{11}+A_{11}^{\prime} P_{1}+\delta_{1}\left\|P_{1}\right\|^{2} I_{n_{1}}+\frac{2 k_{1}^{2}}{\delta_{a}} I_{n_{1}}\right) e_{1} \\
& +e_{2 c}^{\prime}\left(A_{2 c}^{\prime} P_{2}+P_{2} A_{2 c}+\left(\frac{18 \beta^{2}}{\delta_{1}}+\frac{2\left\|C_{2 c}\right\|^{2}}{\delta_{a}}\right) I_{n_{2}+n_{c}}\right) e_{2 c}
\end{aligned}
$$

where $\mathcal{E}=\left(-\alpha \epsilon+\frac{4 \beta^{2}}{\delta_{1}}\right) I_{n}, \mathcal{H}=\alpha P_{a}\left[\begin{array}{c}0 \\ B_{22}\end{array}\right]$ and $\mathcal{J}=-2 W+\delta_{a}\|W\|^{2} I_{m}$.

Therefore, a sufficient condition for global asymptotic stability is for the three terms in the above inequality to all be strictly negative definite. Below, each term is considered in turn.

1.

$$
M_{1}:=\left[\begin{array}{cc}
\left(-\alpha \epsilon+\frac{4 \beta^{2}}{\delta_{1}}\right) I_{n} & \alpha P_{a}\left[\begin{array}{c}
0 \\
B_{22}
\end{array}\right] \\
\star & -2 W+\delta_{a}\|W\|^{2} I_{m}
\end{array}\right]
$$

For any (arbitrarily small) $\delta_{1}$, it is always possible to choose $\alpha$ sufficiently large such that $-\alpha \epsilon+\frac{4 \beta^{2}}{\delta_{1}}<0$. Next, for any $W$ it is possible to choose, $\delta_{a}$ sufficiently small such that $-2 W+\delta_{a}\|W\|^{2}<0$ for any $W>0$. Finally, choosing $W=\alpha_{W} \tilde{W}, \alpha_{W}>0$ is chosen sufficiently large such that $M_{1}<0$.

2.

$$
M_{2}:=P_{1} A_{11}+A_{11}^{\prime} P_{1}+\delta_{1}\left\|P_{1}\right\|^{2} I_{n_{1}}+\frac{k_{1}^{2}}{\delta_{a}} I_{n_{1}}
$$

By Assumption 1 there exists a $\tilde{P}_{1}$ such that $\tilde{P}_{1} A_{11}+A_{11}^{\prime} \tilde{P}_{1}=-\tilde{Q}_{1}<0$. Then with $P_{1}=$ $\alpha_{1} \tilde{P}_{1}$, choosing $\delta_{1}$ sufficiently small ensures that $\alpha_{1}\left(\tilde{P}_{1} A_{11}+A_{11}^{\prime} \tilde{P}_{1}\right)+\alpha_{1}^{2} \delta_{1}\left\|\tilde{P}_{1}\right\|^{2} I_{n_{1}}$ for any $\tilde{P}_{1}$. Next choosing $\alpha_{1}$ sufficiently large ensures that $M_{2}<0$.

3.

$$
M_{3}:=A_{2 c}^{\prime} P_{2}+P_{2} A_{2 c}+\frac{9 \beta^{2}}{\delta_{1}} I_{n_{2}+n_{c}}+\frac{\left\|C_{2 c}\right\|^{2}}{\delta_{a}} I_{n_{2}+n_{c}}
$$


By Assumption 2 there exists a $P_{2}=\alpha_{2} \tilde{P}_{2}$ such that $\alpha_{2}\left(A_{2 c}^{\prime} \tilde{P}_{2}+\tilde{P}_{2} A_{2 c}\right)=-\alpha_{2} Q_{2}<0$. Choosing $\alpha_{2}$ sufficiently large then implies that $M_{3}$ is negative definite for any given $\beta, \delta_{1}, \delta_{a}$ and $C_{2 c}$.

Thus by judicious choice of the free parameters $W, \delta_{a}, \delta_{1}, \alpha, \alpha_{1}$ and $\alpha_{2}$, it is always possible to ensure $\dot{V}_{\text {tot }}(\xi) \leq-\eta\|\xi\|^{2}$ and, thus, the system is exponentially stable.

\subsection{Stability proof of the reduced-order IMC-type AW compensator of Section 3.2}

In this case $x_{a 1} \equiv 0$, so with the notation from the proof of Proposition 1 , it follows that $e_{1} \equiv x_{1}$. The approach taken in the proof is similar to that in Proposition 1 and involves one proving that the system in the coordinates $\left(e_{1}, e_{2}, x_{c}, x_{a 2}\right)$ is exponentially stable.

where $C_{2 c}=\left[\begin{array}{ll}C_{2} D_{c} & C_{c}\end{array}\right]$.

$$
\left\{\begin{aligned}
\dot{e}_{1} & =A_{11} e_{1}+A_{12}\left(x_{1}\right) x_{2} \\
\dot{e}_{2 c} & =A_{2 c} e_{2 c} \\
\dot{x}_{a 2} & =A_{22} x_{a 2}-B_{22} \mathrm{Dz}\left(y_{c}\right) \\
y_{c} & =C_{2 c} e_{2 c}-f_{1}\left(x_{1}\right)
\end{aligned}\right.
$$

8.2.1. Lyapunov function candidate $V_{a 2}\left(x_{a 2}\right)$ : Let $V_{a 2}\left(x_{a 2}\right)=x_{a 2}^{\prime} P_{a 2} x_{a 2}$ with $P_{a 2}>0$. By identical arguments used in the proof of Proposition 1, and using Assumption 1 and Fact 1, it follows that the time-derivative of $V_{a 2}\left(x_{a 2}\right)$ along the $x_{a 2}$ subsystem is bounded by

$$
\begin{aligned}
\dot{V}_{a 2} & \leq x_{a 2}^{\prime}\left(P_{a 2} A_{22}+A_{22}^{\prime} P_{a 2}\right) x_{a 2}+2 x_{a 2}^{\prime} P_{a 2} B_{22} \phi \\
& +2 \phi^{\prime} W[\underbrace{C_{2 c} e_{2 c}-f_{1}\left(x_{1}\right)}_{y_{c} \text { from Eq.37 }}-\phi] \\
& \leq\left[\begin{array}{c}
x_{a 2} \\
\phi
\end{array}\right]^{\prime}\left[\begin{array}{cc}
P_{a 2} A_{22}+A_{22}^{\prime} P_{a 2} & P_{a 2} B_{22} \\
\star & -2 W
\end{array}\right]\left[\begin{array}{c}
x_{a 2} \\
\phi
\end{array}\right] \\
& +2\|\phi\|\|W\|\left(\left\|C_{2 c} e_{2 c}\right\|+k_{1}\left\|e_{1}\right\|\right) \\
& \leq\left[\begin{array}{c}
x_{a 2} \\
\phi
\end{array}\right]^{\prime}\left[\begin{array}{cc}
P_{a 2} A_{22}+A_{22}^{\prime} P_{a 2} & P_{a 2} B_{22} \\
\star & -2 W
\end{array}\right]\left[\begin{array}{c}
x_{a 2} \\
\phi
\end{array}\right] \\
& +\delta_{a}\|W\|^{2}\|\phi\|^{2}+\frac{2}{\delta_{a}}\left(\left\|C_{2 c}\right\|^{2}\left\|e_{2 c}\right\|^{2}+k_{1}^{2}\left\|e_{1}\right\|^{2}\right)
\end{aligned}
$$

where again $W>0$ is some positive definite diagonal matrix and $\delta_{a}>0$ is some positive scalar.

8.2.2. Lyapunov function candidate: $V_{1}\left(e_{1}\right)$ : Let $V_{1}\left(e_{1}\right)=e_{1}^{\prime} P_{1} e_{1}>0$. Then we have, using Assumption 1

$$
\begin{aligned}
\dot{V}\left(e_{1}\right) & \leq e_{1}^{\prime}\left(P_{1} A_{11}+A_{11}^{\prime} P_{1}\right) e_{1}+2\left\|e_{1}\right\|\left\|P_{1}\right\|\left\|A_{12}\left(e_{1}\right) x_{2}\right\| \\
& \leq e_{1}^{\prime}\left(P_{1} A_{11}+A_{11}^{\prime} P_{1}\right) e_{1}+\delta_{1}\left\|P_{1}\right\|^{2}\left\|e_{1}\right\|^{2}+\frac{\beta^{2}}{\delta_{1}}\left\|x_{2}\right\|^{2} \\
& \leq e_{1}^{\prime}\left(P_{1} A_{11}+A_{11}^{\prime} P_{1}\right) e_{1}+\delta_{1}\left\|P_{1}\right\|^{2}\left\|e_{1}\right\|^{2} \\
& +\frac{2 \beta^{2}}{\delta_{1}}\left(\left\|x_{2 c}\right\|^{2}+\left\|x_{a 2}\right\|^{2}\right)
\end{aligned}
$$

where $\delta_{1}>0$ is some positive scalar. 
8.2.3. Lyapunov function candidate $V_{2}\left(e_{2 c}\right)$ :

$$
\dot{V}\left(e_{2 c}\right)=e_{2 c}^{\prime}\left(A_{2 c}^{\prime} P_{2}+P_{2} A_{2 c}\right) e_{2 c}
$$

Sum of Lyapunov function candidates:Collecting terms together as in the proof of Proposition 1 , and defining the state-vector $\xi_{r}:=\left[e_{1}^{\prime}, e_{2 c}^{\prime}, x_{2 a}\right]^{\prime}$, then allows us to write

$$
\begin{aligned}
\dot{V}_{r}\left(\xi_{r}\right)= & \dot{V}_{a 2}\left(x_{a 2}\right)+\dot{V}_{1}\left(e_{1}\right)+\dot{V}_{2}\left(e_{2 c}\right) \\
\leq & {\left[\begin{array}{c}
x_{a} \\
\phi
\end{array}\right]^{\prime} M_{1 r}\left[\begin{array}{c}
x_{a} \\
\phi
\end{array}\right] } \\
& +e_{1}^{\prime}\left(P_{1} A_{11}+A_{11}^{\prime} P_{1}+\delta_{1}\left\|P_{1}\right\|^{2} I_{n_{1}}+\frac{k_{1}^{2}}{\delta_{a}} I_{n_{1}}\right) e_{1} \\
& +e_{2 c}^{\prime}\left(A_{2 c}^{\prime} P_{2}+P_{2} A_{2 c}+\left(\frac{\beta^{2}}{\delta_{1}}+\frac{\left\|C_{2 c}\right\|^{2}}{\delta_{a}}\right) I_{n_{1}+n_{c}}\right) e_{2 c}
\end{aligned}
$$

where

$$
M_{1 r}=\left[\begin{array}{cc}
P_{a 2} A_{22}+A_{22}^{\prime} P_{a 2}+\frac{\beta^{2}}{\delta_{1}} I_{n_{2}} & P_{a 2} B_{22} \\
\star & -2 W+\delta_{a}\|W\|^{2} I_{m}
\end{array}\right]
$$

As in Proposition 1, by Assumption 1 and with judicious choices of the scalars $\delta_{1}, \delta_{a}$ and the matrices $W, P_{a 2}, P_{1}$ and $P_{2}$, it follows that $\dot{V}_{r}\left(\xi_{r}\right) \leq-\eta\left\|\xi_{r}\right\|^{2}$ and exponential stability follows.

\subsection{Stability proof of the improved reduced-order AW compensator of Section 3.3}

The approach taken in the proof is similar to that in Propositions 1,2. The closed-loop interconnection (Fig. 3) of the plant (1), the controller (2) and the anti-windup compensator (9) can be described in the $\left(e_{1}, e_{2}, x_{c}, x_{a 2}\right)$ coordinates (recall $\left.e_{1} \equiv x_{1}\right)$ as follows:

$$
\left\{\begin{aligned}
\dot{e}_{1} & =A_{11} x_{1}+A_{12}\left(x_{1}\right) x_{2} \\
\dot{e}_{2 c} & =A_{2 c} e_{2 c} \\
\dot{x}_{a 2} & =\left(A_{22}+B_{22} F\right) x_{a 2}-B_{22} \mathrm{Dz}\left(y_{c}\right) \\
y_{c} & =C_{2 c} e_{2 c}-f_{1}\left(x_{1}\right)+F x_{a 2}
\end{aligned}\right.
$$

8.3.1. Lyapunov function candidate $V_{a 2}\left(x_{a 2}\right)$ : Let $V_{a 2}\left(x_{a 2}\right)=x_{a 2}^{\prime} P_{a 2} x_{a 2}>0$. Then, similar to the proof of Proposition 1, and using Assumption 1, it follows that

$$
\begin{aligned}
\dot{V}_{a 2} & =2 x_{a 2}^{\prime} P_{a 2}\left[\left(A_{22}+B_{22} F\right) x_{a 2}-B_{22} \phi\right] \\
& \leq x_{a 2}^{\prime}\left[P_{a 2}^{\prime}\left(A_{22}+B_{22} F\right)+\left(A_{22}+B_{22} F\right)^{\prime} P_{a 2}\right] x_{a 2} \\
& -2 x_{a 2}^{\prime} P_{a 2} B_{22} \phi \\
& +2 \phi^{\prime} W[\underbrace{C_{2 c} e_{2 c}-f_{1}\left(x_{1}\right)+F x_{a 2}}_{y_{c} \text { from Eq.45 }}-\phi] \\
& \leq\left[\begin{array}{c}
x_{a 2} \\
\phi
\end{array}\right]^{\prime}\left[\begin{array}{cc}
\mathcal{N} & -P_{a 2} B_{22}+F^{\prime} W \\
\star & -2 W
\end{array}\right]\left[\begin{array}{c}
x_{a 2} \\
\phi
\end{array}\right] \\
& +2\|\phi\|\|W\|\left(\left\|C_{2 c} e_{2 c}-f\left(x_{1}\right)\right\|\right)
\end{aligned}
$$


where $\mathcal{N}=P_{a 2}\left(A_{22}+B_{22} F\right)+\left(A_{22}+B_{22} F\right)^{\prime} P_{a 2}$. Using inequality (26) it follows, for any $\delta_{a}>0$, that

$$
\begin{aligned}
\dot{V}\left(x_{a}\right) & \leq\left[\begin{array}{c}
x_{a 2} \\
\phi
\end{array}\right]^{\prime}\left[\begin{array}{cc}
\mathcal{N} & -P_{a 2} B_{22}+F^{\prime} W \\
\star & -2 W
\end{array}\right]\left[\begin{array}{c}
x_{a 2} \\
\phi
\end{array}\right] \\
& +\delta_{a}\|\phi\|^{2}\|W\|^{2}+\frac{1}{\delta_{a}}\left\|C_{2 c} e_{2 c}-f\left(x_{1}\right)\right\|^{2} \\
& \leq\left[\begin{array}{c}
x_{a 2} \\
\phi
\end{array}\right]^{\prime}\left[\begin{array}{cc}
\mathcal{N} & -P_{a 2} B_{22}+F^{\prime} W \\
\star & -2 W
\end{array}\right]\left[\begin{array}{c}
x_{a 2} \\
\phi
\end{array}\right] \\
& +\delta_{a}\|\phi\|^{2}\|W\|^{2}+\frac{2}{\delta_{a}}\left\|C_{2 c}\right\|^{2}\left\|e_{2 c}\right\|^{2}+\frac{2}{\delta_{a}} k_{1}^{2}\left\|x_{1}\right\|^{2}
\end{aligned}
$$

where $W>0$ is some positive definite diagonal matrix and $\delta_{a}>0$ is some positive scalar.

8.3.2. Lyapunov function candidate $V_{1}\left(e_{1}\right)$ : Let $V_{1}\left(e_{1}\right)=e_{1}^{\prime} P_{1} e_{1}>0$. Then we have, using Assumption 1

$$
\begin{aligned}
\dot{V}\left(e_{1}\right) & =2 e_{1}^{\prime} P_{1}\left(A_{11} e_{1}+A_{12}\left(e_{1}\right) x_{2}\right) \\
& =2 e_{1}^{\prime} P_{1} A_{11} e_{1}+2 e_{1}^{\prime} P_{1} A_{12}\left(e_{1}\right) x_{2} \\
& \leq e_{1}^{\prime}\left(P_{1} A_{11}+A_{11}^{\prime} P_{1}\right) e_{1}+2\left\|e_{1}\right\|\left\|P_{1}\right\|\left\|A_{12}\left(e_{1}\right) x_{2}\right\| \\
& \leq e_{1}^{\prime}\left(P_{1} A_{11}+A_{11}^{\prime} P_{1}\right) e_{1}+2\left\|e_{1}\right\|\left\|P_{1}\right\|\left\|A_{12}\left(e_{1}\right)\right\|\left\|x_{2}\right\|
\end{aligned}
$$

Using Young's inequality (26)

$$
\dot{V}\left(e_{1}\right) \leq e_{1}^{\prime}\left(P_{1} A_{11}+A_{11}^{\prime} P_{1}\right) e_{1}+\delta_{1}\left\|P_{1}\right\|^{2}\left\|e_{1}\right\|^{2}+\frac{\beta^{2}}{\delta_{1}}\left\|x_{2}\right\|^{2}
$$

where $\delta_{1}>0$ is some positive scalar. Now $e_{2}=x_{2}-x_{a 2} \Rightarrow x_{2}=e_{2}-x_{a 2}$, hence

$$
\begin{aligned}
\dot{V}\left(e_{1}\right) & \leq e_{1}^{\prime}\left(P_{1} A_{11}+A_{11}^{\prime} P_{1}\right) e_{1}+\delta_{1}\left\|P_{1}\right\|^{2}\left\|e_{1}\right\|^{2} \\
& +\frac{2 \beta^{2}}{\delta_{1}}\left(\left\|e_{2}\right\|^{2}+\left\|x_{a 2}\right\|^{2}\right)
\end{aligned}
$$

8.3.3. Lyapunov function candidate $V_{2}\left(e_{2 c}\right)$ : As before $V_{2}\left(e_{2 c}\right)=e_{2 c}^{\prime} P_{2} e_{2 c}>0$. Then we have, using Assumption 2,

$$
\dot{V}\left(e_{2 c}\right)=e_{2 c}^{\prime}\left(A_{2 c}^{\prime} P_{2}+P_{2} A_{2 c}\right) e_{2 c}
$$

Sum of Lyapunov function candidates.Collecting terms together and defining the state-vector $\xi_{r o}:=\left[e_{1}^{\prime}, e_{2 c}^{\prime}, x_{2 a}\right]^{\prime}$, then allows us to write $V_{t o t}\left(\xi_{r o}\right)=V_{a 2}\left(x_{a 2}\right)+V_{1}\left(e_{1}\right)+V_{2}\left(e_{2 c}\right)$ 


$$
\begin{aligned}
\dot{V}_{\text {tot }}\left(\xi_{\text {ro }}\right)= & {\left[\begin{array}{c}
x_{a 2} \\
\phi
\end{array}\right]^{\prime}\left[\begin{array}{cc}
\mathcal{N} & -P_{a 2} B_{22}+F^{\prime} W \\
\star & -2 W+\delta_{a}\|W\|^{2}
\end{array}\right]\left[\begin{array}{c}
x_{a 2} \\
\phi
\end{array}\right] } \\
& +\frac{2}{\delta_{a}}\left\|C_{2 c}\right\|^{2}\left\|e_{2 c}\right\|^{2}+\frac{2}{\delta_{a}} k_{1}\left\|e_{1}\right\|^{2} \\
& +e_{1}^{\prime}\left(P_{1} A_{11}+A_{11}^{\prime} P_{1}+\delta_{1}\left\|P_{1}\right\|^{2}\right) e_{1} \\
+ & \frac{2 \beta^{2}}{\delta_{1}}\left(\left\|e_{2 c}\right\|^{2}+\left\|x_{a 2}\right\|^{2}\right) \\
& +e_{2 c}^{\prime}\left(P_{2} A_{2 c}+A_{2 c}^{\prime} P_{2}\right) e_{2 c} \\
= & {\left[\begin{array}{c}
x_{a 2} \\
\phi
\end{array}\right]^{\prime}\left[\begin{array}{ll}
\mathcal{N} & -P_{a 2} B_{22}+F^{\prime} W \\
\star & -2 W+\delta_{a}\|W\|^{2}
\end{array}\right]\left[\begin{array}{c}
x_{a 2} \\
\phi
\end{array}\right] } \\
& +e_{1}^{\prime}\left(P_{1} A_{11}+A_{11}^{\prime} P_{1}+\delta_{1}\left\|P_{1}\right\|^{2} I_{n_{1}}+\frac{2}{\delta_{a}} k_{1}^{2} I_{n_{1}}\right) e_{1} \\
& +e_{2 c}^{\prime}\left(P_{2} A_{2 c}+A_{2 c}^{\prime} P_{2}+\frac{2}{\delta_{a}}\left\|C_{2 c}\right\|^{2} I_{n_{2}+n_{c}}\right. \\
+ & \left.\frac{2 \beta^{2}}{\delta_{1}} I_{n_{2}+n_{c}}\right) e_{2 c}
\end{aligned}
$$

For stability it is sufficient for each of the three terms in the above inequality to be negative definite. Consider each term individually.

1.

$$
M_{1 \text { ro }}:=\left[\begin{array}{cc}
\mathcal{N}+\frac{2 \beta^{2}}{\delta_{1}} I_{n_{2}} & -P_{a 2} B_{22}+F^{\prime} W \\
\star & -2 W+\delta_{a}\|W\|^{2}
\end{array}\right]
$$

Let's $P_{a 2}=\alpha \tilde{P}_{a}, W=\alpha \tilde{W}$ and we have

$$
\begin{aligned}
M_{1 \text { ro }} & =\alpha\left[\begin{array}{cc}
\mathcal{Y} & -\tilde{P}_{a} B_{22}+F^{\prime} \tilde{W} \\
\star & -2 \tilde{W}
\end{array}\right] \\
& +\left[\begin{array}{cc}
\frac{2 \beta^{2}}{\delta_{1}} & 0 \\
0 & \delta_{a} \alpha^{2}\|\tilde{W}\|
\end{array}\right]
\end{aligned}
$$

where $\mathcal{Y}=\tilde{P}_{a}\left(A_{22}+B_{22} F\right)+\left(A_{22}+B_{22} F\right)^{\prime} \tilde{P}_{a}$. For large enough $\alpha$ and small enough $\delta_{a}$ this holds for any (arbitrarily small) $\delta_{1}$ provided

$$
\left[\begin{array}{cc}
\mathcal{Y} & -\tilde{P}_{a} B_{22}+F^{\prime} \tilde{W} \\
\star & -2 \tilde{W}
\end{array}\right]<0
$$

Note that Eq. (59) is a bilinear matrix inequality. Using the congruence transformation with $\operatorname{diag}\left(\tilde{P}_{a}^{-1}, \tilde{W}^{-1}\right)=\operatorname{diag}(\tilde{Q}, \tilde{U})$, it is easy to see that eq. (59) is equivalent to

$$
\left[\begin{array}{cc}
A_{22} \tilde{Q}+\tilde{Q} A_{22}^{\prime} & +B_{22} \tilde{L}+\tilde{L}^{\prime} B_{22} \\
\star & -B_{22} \tilde{U}+\tilde{L} \\
\star & -2 \tilde{U}
\end{array}\right]<0
$$

which is an LMI in $\tilde{Q}, \tilde{U}$ and $\tilde{L}=F \tilde{Q}$. 
2.

$$
M_{2 r_{o}}:=P_{1} A_{11}+A_{11}^{\prime} P_{1}+\left(\delta_{1}\left\|P_{1}\right\|^{2}+\frac{2}{\delta_{a}} k_{1}^{2}\right) I_{n_{1}}
$$

With $P_{1}=\alpha_{1} \tilde{P}_{1}$ we have

$$
\begin{aligned}
M_{2 r o} & :=\alpha_{1}\left(\tilde{P}_{1} A_{11}+A_{11}^{\prime} \tilde{P}_{1}\right) \\
& +\left(\alpha_{1}^{2} \delta_{1}\left\|\tilde{P}_{1}\right\|^{2}+\frac{2}{\delta_{a}} k_{1}^{2}\right) I_{n_{1}}
\end{aligned}
$$

Now by choosing $\alpha_{1}$ sufficiently large and $\delta_{1}$ sufficiently small $M_{2 r_{o}}$ is negative definite for any (arbitrarily small) $\delta_{a}$.

3.

$$
M_{3 r o}:=P_{2} A_{2 c}+A_{2 c}^{\prime} P_{2}+\left(\frac{2}{\delta_{a}}\left\|C_{2 c}\right\|^{2}+\frac{2 \beta^{2}}{\delta_{1}}\right) I_{n_{2}+n_{c}}
$$

Choosing $P_{2}=\alpha_{2} \tilde{P}_{2}$, we have

$$
\begin{aligned}
M_{3 r o} & =\alpha_{2}\left(\tilde{P}_{2} A_{2 c}+A_{2 c}^{\prime} \tilde{P}_{2}\right) \\
& +\left(\frac{2}{\delta_{a}}\left\|C_{2 c}\right\|^{2}+\frac{2 \beta^{2}}{\delta_{1}}\right) I_{n_{2}+n_{c}}
\end{aligned}
$$

So for any arbitrarily small $\delta_{a}, \delta_{1}, \alpha_{2}$ can always be chosen so that $M_{3 r o}$ is negative definite. 
Table 1: AW schemes.

\begin{tabular}{|c|c|c|c|c|c|c|c|c|}
\hline Wave profiles & - & no saturation & saturation & NL IMC & NL RO & L IMC & L2 decoupled RO & decay rate $\mathrm{RO}$ \\
\hline \multirow{3}{*}{$\begin{array}{l}\mathrm{H}=1.0 \mathrm{~m}, \\
\mathrm{~T} 1=10 \mathrm{~s}\end{array}$} & $\mathrm{PO}$ & $3.06 \%$ & $27.08 \%$ & stable $^{[3]}$ & stable ${ }^{[3]}$ & stable ${ }^{[3]}$ & $1.08 \%$ & $1.08 \%$ \\
\hline & ST & $97 \mathrm{sec}$ & - & - & - & - & $387 \mathrm{sec}$ & $387 \mathrm{sec}$ \\
\hline & FV & $6 \cdot 10^{6} P_{a}$ & no tracking & - & - & - & $6 \cdot 10^{6} P_{a}$ & $6 \cdot 10^{6} P_{a}$ \\
\hline \multirow{3}{*}{$\begin{array}{l}\mathrm{H}=1.0 \mathrm{~m}, \\
\mathrm{~T} 1=10 \mathrm{~s} \\
(\mathrm{v} 2)\end{array}$} & $\mathrm{PO}$ & $3.06 \%$ & $28.2 \%$ & stable ${ }^{[3]}$ & stable ${ }^{[3]}$ & stable $^{[3]}$ & $0 \%$ & $0 \%$ \\
\hline & ST & $97 \mathrm{sec}$ & $990 \mathrm{sec}$ & - & - & - & $441 \mathrm{sec}$ & $441 \mathrm{sec}$ \\
\hline & FV & $6 \cdot 10^{6} P_{a}$ & around $6 \cdot 10^{6} P_{a}$ & - & - & - & $6 \cdot 10^{6} P_{a}$ & $6 \cdot 10^{6} P_{a}$ \\
\hline \multirow{3}{*}{$\begin{array}{l}\mathrm{H}=1.0 \mathrm{~m}, \\
\mathrm{~T} 1=10 \mathrm{~s} \\
(\mathrm{v} 3)\end{array}$} & PO & $3.06 \%$ & $27.21 \%$ & stable ${ }^{[3]}$ & stable ${ }^{[3]}$ & stable $^{[3]}$ & $0 \%$ & $0 \%$ \\
\hline & $\mathrm{ST}$ & $97 \mathrm{sec}$ & $980 \mathrm{sec}$ & - & - & - & $429 \mathrm{sec}$ & $429 \mathrm{sec}$ \\
\hline & FV & $6 \cdot 10^{6} P_{a}$ & around $6 \cdot 10^{6} P_{a}$ & - & - & - & $6 \cdot 10^{6} P_{a}$ & $6 \cdot 10^{6} P_{a}$ \\
\hline \multirow{3}{*}{$\begin{array}{l}\mathrm{H}=1.1 \mathrm{~m} \\
\mathrm{~T} 1=10 \mathrm{~s}\end{array}$} & $\mathrm{PO}$ & $3.06 \%$ & $19.28 \%$ & stable ${ }^{[3]}$ & stable ${ }^{[3]}$ & stable $^{[3]}$ & $0 \%$ & $0 \%$ \\
\hline & ST & $97 \mathrm{sec}$ & $648 \mathrm{sec}$ & - & - & - & $298 \mathrm{sec}$ & $298 \mathrm{sec}$ \\
\hline & FV & $6 \cdot 10^{6} P_{a}$ & around $6 \cdot 10^{6} P_{a}$ & - & - & - & $6 \cdot 10^{6} P_{a}$ & $6 \cdot 10^{6} P_{a}$ \\
\hline \multirow{3}{*}{$\begin{array}{l}\mathrm{H}=1.1 \mathrm{~m}, \\
\mathrm{~T} 1=10 \mathrm{~s} \\
(\mathrm{v} 2)\end{array}$} & $\mathrm{PO}$ & $3.06 \%$ & $30.11 \%$ & stable ${ }^{[3]}$ & stable $^{[3]}$ & stable $^{[3]}$ & $18.01 \%$ & $18.01 \%$ \\
\hline & ST & $97 \mathrm{sec}$ & no steady-state & - & - & - & $176 \mathrm{sec}$ & $176 \mathrm{sec}$ \\
\hline & $\mathrm{FV}$ & $6 \cdot 10^{6} P_{a}$ & outside limits & - & - & - & around $6 \cdot 10^{6} P_{a}$ & around $6 \cdot 10^{6} P_{a}$ \\
\hline \multirow{3}{*}{$\begin{array}{l}\mathrm{H}=1.1 \mathrm{~m}, \\
\mathrm{~T} 1=10 \mathrm{~s} \\
(\mathrm{v} 3)\end{array}$} & $\mathrm{PO}$ & $3.06 \%$ & $22.61 \%$ & stable ${ }^{[3]}$ & stable ${ }^{[3]}$ & stable $\mathrm{e}^{[3]}$ & $4.1 \%$ & $4.1 \%$ \\
\hline & ST & $97 \mathrm{sec}$ & $565 \mathrm{sec}$ & - & - & - & $199.5 \mathrm{sec}$ & $199.5 \mathrm{sec}$ \\
\hline & $\mathrm{FV}$ & $6 \cdot 10^{6} P_{a}$ & around $6 \cdot 10^{6} P_{a}$ & - & - & - & $6 \cdot 10^{6} P_{a}$ & $6 \cdot 10^{6} P_{a}$ \\
\hline \multirow{3}{*}{$\begin{array}{l}\mathrm{H}=1.2 \mathrm{~m} \\
\mathrm{~T} 1=10 \mathrm{~s}\end{array}$} & $\mathrm{PO}$ & $3.06 \%$ & $19.66 \%$ & stable ${ }^{[3]}$ & stable ${ }^{[3]}$ & stable ${ }^{[3]}$ & $7.78 \%$ & $7.78 \%$ \\
\hline & ST & $97 \mathrm{sec}$ & $413 \mathrm{sec}$ & - & - & - & $193 \mathrm{sec}$ & $193 \mathrm{sec}$ \\
\hline & $\mathrm{FV}$ & $6 \cdot 10^{6} P_{a}$ & around $6 \cdot 10^{6} P_{a}$ & - & - & - & around $6 \cdot 10^{6} P_{a}$ & around $6 \cdot 10^{6} P_{a}$ \\
\hline \multirow{3}{*}{$\begin{array}{l}\mathrm{H}=1.2 \mathrm{~m}, \\
\mathrm{~T} 1=10 \mathrm{~s} \\
(\mathrm{v} 2)\end{array}$} & $\mathrm{PO}$ & $3.06 \%$ & $11.83 \%$ & stable ${ }^{[3]}$ & stable ${ }^{[3]}$ & stable ${ }^{[3]}$ & $2.53 \%$ & $2.53 \%$ \\
\hline & $\mathrm{ST}$ & $97 \mathrm{sec}$ & $371 \mathrm{sec}$ & - & - & - & $170 \mathrm{sec}$ & $170 \mathrm{sec}$ \\
\hline & $\mathrm{FV}$ & $6 \cdot 10^{6} P_{a}$ & around $6 \cdot 10^{6} P_{a}$ & - & - & - & $6 \cdot 10^{6} P_{a}$ & $6 \cdot 10^{6} P_{a}$ \\
\hline \multirow{3}{*}{$\begin{array}{l}\mathrm{H}=1.2 \mathrm{~m} \\
\mathrm{~T} 1=10 \mathrm{~s} \\
(\mathrm{v} 3)\end{array}$} & $\mathrm{PO}$ & $3.06 \%$ & $14.38 \%$ & stable ${ }^{[3]}$ & stable ${ }^{[3]}$ & stable ${ }^{[3]}$ & $6.98 \%$ & $6.98 \%$ \\
\hline & ST & $97 \mathrm{sec}$ & $365 \mathrm{sec}$ & - & - & - & $156 \mathrm{sec}$ & $156 \mathrm{sec}$ \\
\hline & $\mathrm{FV}$ & $6 \cdot 10^{6} P_{a}$ & around $6 \cdot 10^{6} P_{a}$ & - & - & - & $6 \cdot 10^{6} P_{a}$ & $6 \cdot 10^{6} P_{a}$ \\
\hline \multirow{3}{*}{$\begin{array}{l}H=1.3 \mathrm{~m} \\
\mathrm{~T} 1=10 \mathrm{~s}\end{array}$} & $\mathrm{PO}$ & $3.06 \%$ & $20.98 \%$ & stable $^{[3]}$ & stable $^{[3]}$ & stable ${ }^{[3]}$ & $13.3 \%$ & $13.3 \%$ \\
\hline & ST & $97 \mathrm{sec}$ & oscillatory & - & - & - & $189 \mathrm{sec}$ & $189 \mathrm{sec}$ \\
\hline & $\mathrm{FV}$ & $6 \cdot 10^{6} P_{a}$ & out of limits & - & - & - & around $6 \cdot 10^{6} P_{a}$ & around $6 \cdot 10^{6} P_{a}$ \\
\hline \multirow{3}{*}{$\begin{array}{l}H=1.3 \mathrm{~m}, \\
\mathrm{~T} 1=10 \mathrm{~s} \\
(\mathrm{v} 2)\end{array}$} & $\mathrm{PO}$ & $3.06 \%$ & $8.95 \%$ & stable ${ }^{[3]}$ & stable $^{[3]}$ & stable $\mathrm{e}^{[3]}$ & $6.66 \%$ & $6.66 \%$ \\
\hline & $\mathrm{ST}$ & $97 \mathrm{sec}$ & $114 \mathrm{sec}$ & - & - & - & $109 \mathrm{sec}$ & $109 \mathrm{sec}$ \\
\hline & $\mathrm{FV}$ & $6 \cdot 10^{6} P_{a}$ & around $6 \cdot 10^{6} P_{a}$ & - & - & - & $6 \cdot 10^{6} P_{a}$ & $6 \cdot 10^{6} P_{a}$ \\
\hline \multirow{3}{*}{$\begin{array}{l}H=1.3 \mathrm{~m} \\
\mathrm{~T} 1=10 \mathrm{~s} \\
(\mathrm{v} 3)\end{array}$} & $\mathrm{PO}$ & $3.06 \%$ & $11.43 \%$ & stable $^{[3]}$ & stable $^{[3]}$ & stable $\mathrm{e}^{[3]}$ & $5.83 \%$ & $5.83 \%$ \\
\hline & $\mathrm{ST}$ & $97 \mathrm{sec}$ & no steady-state & - & - & - & $155 \mathrm{sec}$ & $155 \mathrm{sec}$ \\
\hline & $\mathrm{FV}$ & $6 \cdot 10^{6} P_{a}$ & out of limits & - & - & - & $6 \cdot 10^{6} P_{a}$ & $6 \cdot 10^{6} P_{a}$ \\
\hline
\end{tabular}

${ }^{1} \mathrm{PO}=$ Peak Overshoot, $\mathrm{ST}=$ Settling Time, FV = Final Value, NL = nonlinear, $\mathrm{L}=$ linear, $\mathrm{RO}=$ reduced-order.

${ }^{2}$ When compensators have simply maintained stability but have provided no tracking performance, they are labelled "stable" 